\title{
Contrast Gain Control in the Visual Cortex: Monocular Versus Binocular Mechanisms
}

\author{
Anthony M. Truchard, Izumi Ohzawa, and Ralph D. Freeman \\ Group in Vision Science, School of Optometry, University of California, Berkeley, California 94720-2020
}

In this study, we compare binocular and monocular mechanisms underlying contrast encoding by binocular simple cells in primary visual cortex. At mid to high levels of stimulus contrast, contrast gain of cortical neurons typically decreases as stimulus contrast is increased (Albrecht and Hamilton, 1982). We have devised a technique by which it is possible to determine the relative contributions of monocular and binocular processes to such reductions in contrast gain. First, we model the simple cell as an adjustable linear mechanism with a static output nonlinearity. For binocular cells, the linear mechanism is sensitive to inputs from both eyes. To constrain the parameters of the model, we record from binocular simple cells in striate cortex. To activate each cell, drifting sinusoidal gratings are presented dichoptically at various relative interocular phases. Stimulus contrast for one eye is varied over a large range whereas that for the other eye is fixed. We then determine the best-fitting parameters of the model for each cell for all of the interocular contrast ratios. This allows us to determine the effect of contrast on the contrast gain of the system. Finally, we decompose the contrast gain into monocular and binocular components. Using the data to constrain the model for a fixed contrast in one eye and increased contrasts in the other eye, we find steep reductions in monocular gain, whereas binocular gain exhibits modest and variable changes. These findings demonstrate that contrast gain reductions occur primarily at a monocular site, before convergence of information from the two eyes.

Key words: contrast gain control; simple cells; striate cortex; binocular vision; cat; nonlinearity
Multiple gain control mechanisms participate in the encoding of visual information by the brain. In the retina, luminance gain control helps the visual system cope with the $10^{10}$-fold range of luminance levels observed in natural scenes (Shapley and EnrothCugell, 1984). A consequence of luminance gain control, along with the well known center-surround organization of retinal ganglion cells, is that neural responses depend more on local image contrast than on the absolute average luminance level. Näively, this suggests one possible model of contrast encoding: responses of visual neurons could increase in linear proportion to image contrast. However, in primary visual cortex, neuronal response exhibits compression and saturation as contrast of a stimulus is increased (Albrecht and Hamilton, 1982), a finding that can be explained in terms of a contrast gain control system (Albrecht and Geisler, 1991; Heeger, 1992b).

Contrast gain control has been demonstrated at both retinal (Shapley and Victor, 1978; Benardete et al., 1992) and cortical levels. For example, in cortical area 17, short-term contrast adaptation $(\sim 5 \mathrm{sec})$ produces a steep contrast-response curve centered around the contrast adaptation level, an effect not seen in the lateral geniculate nucleus (Ohzawa et al., 1982, 1985). Contrast gain control is consistent with the preservation of response selectivity in the visual cortex at high stimulus contrasts (Albrecht and Hamilton, 1982; Sclar and Freeman, 1982; Albrecht and Geisler, 1991; Heeger, 1992b), and models of gain control (Albrecht and Geisler, 1991; Heeger, 1992a,b, 1993; Carandini et al.,

\footnotetext{
Received Sept. 21, 1999; revised Feb. 4, 2000; accepted Feb. 7, 2000.

This work was supported by National Eye Institute Research and CORE Grants (EY-01175, EY-12393, and EY-03176) and by a National Science Foundation Graduate Research Fellowship to A.M.T.

Correspondence should be addressed to Ralph D. Freeman, University of California, School of Optometry, 360 Minor Hall \#2020, Berkeley, CA 94720-2020. E-mail: freeman@pinoko.berkeley.edu.

Copyright (C) 2000 Society for Neuroscience $\quad 0270-6474 / 00 / 203017-16 \$ 15.00 / 0$
}

1997) are consistent with various experimental results (Heeger, 1992b, 1993; Tolhurst and Heeger, 1997a,b; Carandini et al., 1997; Nestares and Heeger, 1997). A number of suppressive effects in area 17 may be involved in or related to contrast gain control; these include cross-orientation inhibition (Morrone et al., 1982; DeAngelis et al., 1992; Walker et al., 1998), spatial frequencyspecific inhibition (DeValois and Tootell, 1983; Bauman and Bonds, 1991), surround suppression (DeAngelis et al., 1994), and interocular suppression (Sengpiel and Blakemore, 1994; Sengpiel et al., 1995).

To identify the functional site of contrast gain control, it is necessary to know whether adjustments in contrast gain occur before or after convergence of information from the two eyes. There have been demonstrations of interocular suppression (Sengpiel et al., 1995) and interocular transfer of contrast adaptation effects (Sclar et al., 1985; Maffei et al., 1986; Marlin et al., 1991). Thus certain stimulus-dependent gain reductions may be mediated at a binocular site in area 17 . However, we have reported previously (Freeman and Ohzawa, 1990; Ohzawa and Freeman, 1994) that changes in relative levels of contrast in the two eyes have little effect on the tuning of binocular neurons to interocular phase disparity. This is suggestive of independent monocular gain control for the two eyes, because otherwise the difference in contrast levels would have more greatly disrupted the tuning functions (Ohzawa and Freeman, 1994).

In the current study, we apply a technique that allows us to determine the relative contributions of monocular and binocular mechanisms to contrast gain control. First, we model the cortical simple cell as a linear filter with a static output nonlinearity. Second, to constrain the parameters of the model, we record from binocular simple cells in the cat's striate cortex. To activate each cell, drifting sinusoidal gratings are presented dichoptically at various relative interocular phases. Stimulus contrast for one eye 
is varied over a large range whereas that for the other eye is fixed. For each contrast level, best-fitting monocular and binocular gain parameters are determined; these reflect aggregate transfer characteristics at monocular and binocular sites and may be viewed as gain settings specified by monocular and binocular gain control mechanisms. Experimentally, we find that independent monocular changes in contrast over a 10 -fold or greater range induce a sharp reduction of monocular gain but have little effect on binocular gain. This means that most of the contrast-dependent gain reduction occurs in monocular pathways.

\section{MATERIALS AND METHODS}

Physiological preparation. Standard physiological techniques were used to make extracellular recordings from binocular simple cells in cat striate cortex. Briefly, acepromazine maleate $(0.5 \mathrm{mg} / \mathrm{kg})$ and atropine sulfate $(0.06 \mathrm{mg} / \mathrm{kg})$ were administered $30 \mathrm{~min}$ before anesthesia by halothane $(1.5-2.5 \%)$ or fluothane $(2-3 \%)$. Venous catheters were inserted for drug and fluid drip administration. A tracheostomy was performed, and a tracheal tube was inserted. The head was held in place by a stereotaxic device, and a craniotomy and duratomy were performed at HorseleyClarke P4, near the midline. During recording, paralysis and anesthesia were maintained with an intravenous infusion consisting of gallamine triethiodide (Flaxedil; $10 \mathrm{mg} \cdot \mathrm{kg}^{-1} \cdot \mathrm{hr}^{-1}$ ) and thiamylal sodium (Bio-tal; $\left.0.8 \mathrm{mg} \cdot \mathrm{kg}^{-1} \cdot \mathrm{hr}^{-1}\right)$ in a $5 \%$ dextrose and lactated Ringer's solution $(0.5$ $\left.\mathrm{ml} \cdot \mathrm{kg}^{-1} \cdot \mathrm{hr}^{-1}\right)$. Lactated Ringer's $\left(10 \mathrm{ml} \cdot \mathrm{kg}^{-1} \cdot \mathrm{hr}^{-1}\right)$ was inf used via a drip system to maintain hydration. Ventilation was artificially sustained, using a mixture of $70 \% \mathrm{O}_{2}$ and $30 \% \mathrm{NO}_{2}$, and the respirator's stroke volume was regulated to maintain peak expired $\mathrm{CO}_{2}$ at $4-5 \%$. To retract the nictitating membranes and dilate the pupils, $1 \%$ atropine sulfate and $5 \%$ phenylephrine hydrochloride were applied to the eyes. Contact lenses ( +2 D) with 3-mm-diameter pupils were inserted, and the location of the optic disk was plotted by means of a reversible ophthalmoscope. EEG and EKG were monitored, and temperature was maintained at $\sim 38^{\circ} \mathrm{C}$.

Tungsten electrodes (A-M Systems) or tungsten-in-glass electrodes (Levick, 1972) were used to record from neurons in primary visual cortex. Manually controlled sinusoidal gratings and bars of light were used to make an initial assessment of the receptive fields of responsive neurons. For subsequent study of the cell, we recorded the responses of neurons to drifting sinusoidal gratings presented in one or both eyes. Although the pair of stimulus monitors used for the first two cats was not the same as the pair used for the remaining cats, the display characteristics were similar (mean luminance, $31-45 \mathrm{~cd} / \mathrm{m}^{2}$; refresh rate, $76 \mathrm{~Hz}$; screen size, $28 \times 22 \mathrm{~cm}$; resolution, $1024 \times 804$ pixels $)$. Images from the two monitors were reflected onto the two eyes by a pair of beam splitters ( $70 \%$ reflectance) orientated $\sim 45^{\circ}$ relative to the visual axis of each eye. Contrast is defined as $100 \% \times\left(l u m_{\max }-l u m_{\min }\right) /\left(l u m_{\max }+l u m_{\min }\right)$, where lum $_{\max }$ and $l u m_{\min }$ denote maximum and minimum luminance levels of the sinusoid. For all but the first two cats, calibration data obtained with a Chroma Meter CS-100 (Minolta) was used post hoc to determine the actual values of $l u m_{\min }$ and $l u m_{\max }$ and therefore the actual contrast of the stimuli. Results obtained from the two pairs of monitors are similar and are combined in this study

Sinusoidal gratings were presented for $4 \mathrm{sec}$, with a temporal frequency of $2 \mathrm{~Hz}$. The responses of the neurons were Fourier-analyzed to determine the stimulus-induced increase in mean firing rate (the $F 0$ response) and the first Fourier harmonic of the response at the stimulation frequency (the $F 1$ response). Optimal orientation and spatial frequency were determined separately for the two eyes, based on initial orientation and spatial frequency trials. These optimal values were used for subsequent stimulation of the cell. Cells were classified as simple cells based on classical characteristics (Hubel and Wiesel, 1962) and on a comparison between the amplitudes of the $F 0$ and $F 1$ responses.

For trials in the main experimental blocks, gratings were presented simultaneously to both eyes. Contrast in one eye (the fixed eye) was held at a high value $(48-51 \%)$ or a lower value $(4-10 \%)$, whereas contrast in the other eye (the varied eye) was varied over a 10 -fold or greater range across trials (see Fig. 1 $A$ ). The spatiotemporal phase of the varied-eye grating was also varied, resulting in changes in the relative stimulus phase of the two gratings. Thus, these blocks of trials were two-dimensional matrices, with five or more contrast levels and eight or more relative phase values. Monocular control trials were also included. For each neuron, the data from up to four such blocks contribute to our data analysis. This is because contrast could be varied in either the left or right eye, and in the other eye, contrast could be fixed at either a high or a low level.

Trials within a block were repeated four to five times, and for statistical purposes these repetitions were regarded as independent events. Because eight cycles of response occurred per repetition, there was a total of 32-40 response cycles for a given trial. These response cycles were averaged to produce a peristimulus-time histogram (PSTH), which was then Fourier-analyzed to determine the $F 1$ response.

Data analysis. Using the Levenberg-Marquardt algorithm (Press et al., 1992), a least square fit was made between the neural responses to a block of trials and the quantitative predictions of a parametric model of contrast gain control. Specifically, least square fits were made between model predictions and both the real and imaginary parts of the $F 1$ response. Thus, the fitting procedure effectively made use of both the amplitude and phase of the $F 1$ responses. Unless stated otherwise, the fits did not take into account either the estimated response variance of individual neurons or the covariance that may exist between the real and imaginary components of individual data points. However, control procedures that took response variance and covariance into account produced similar results (see Results).

Because the model of simple cells used in this study is predominantly linear, we were able to use linear regression methods to provide the Levenberg-Marquardt method with reasonable starting conditions for its search. For models that contain a variable offset parameter, the initial value of the offset was set to 0 , and for models with a variable exponent parameter, the initial value was set to 2 . The estimated covariance matrix calculated by the Levenberg-Marquardt algorithm was used to determine $\mathrm{SE}$ values for the model parameters. These are shown in individual figures.

\section{RESULTS}

\section{Simple cell model}

We modeled the simple cell as a system with three components: a linear mechanism, a static output nonlinearity, and one or more gain control mechanisms. This three-component model is not specific to this article; for a recent review, see Carandini et al. (1999). Both intracellular (Ferster, 1988) and extracellular (Movshon et al., 1978; Jones and Palmer, 1987; DeAngelis et al., 1993) studies of simple cells have supported the view that simple cells are predominantly linear. Furthermore, simple linear summation of left- and right-eye inputs can account for the basic features of binocular interaction in simple cells (Ohzawa and Freeman, 1986; Ohzawa et al., 1996). However, simple cells have response thresholds, and this or a rectified power-law nonlinearity is required to explain their behavior (Ohzawa and Freeman, 1986; Albrecht and Geisler, 1991; Heeger, 1992a). Furthermore, for monocularly viewed stimuli, a change in contrast can result in an advance in response phase (Dean and Tolhurst, 1986; Carandini and Heeger, 1994) and a change in contrast gain (Albrecht and Hamilton, 1982). Therefore, any realistic model of a simple cell must allow response phase and gain to change as a function of stimulus contrast.

The details of the model are as follows. The input to each eye (denoted $\mathrm{V}$ and $\mathrm{F}$, for varied eye and fixed eye, respectively) is represented by a single complex number $\left(S_{\mathrm{V}}\right.$ and $S_{\mathrm{F}}$, respectively); this specifies the amplitude (\% contrast) and phase of the sinusoidal grating. For example, $S_{\mathrm{V}}=C_{\mathrm{V}} \mathrm{e}^{2 \pi \cdot \theta_{\mathrm{V}}}$, where $\mathrm{C}_{\mathrm{V}}$ is the contrast level and $\theta_{\mathrm{V}}$ is the spatiotemporal phase in the varied eye. Mathematically, the linear mechanism of the simple cell can be described as the sum of left-eye and right-eye filters. The transfer characteristic of each filter is given by a single complex number $\left(L_{\mathrm{V}}\right.$ or $\left.L_{\mathrm{F}}\right)$, the amplitude and phase of which specify the gain and phase lag of the filter. The quantities $S_{\mathrm{V}} \times L_{\mathrm{V}}$ and $S_{\mathrm{F}} \times$ 
$\mathbf{A}$
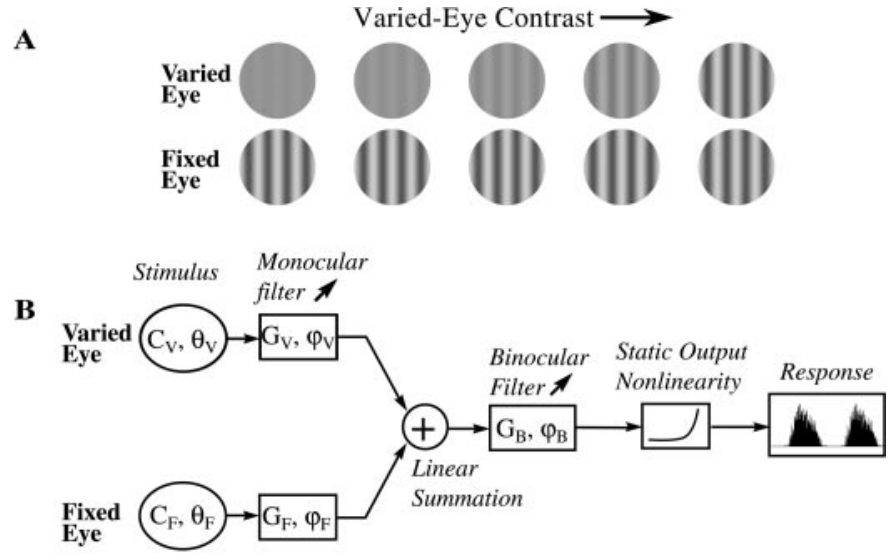

Figure 1. A, Experimental design. In each trial, drifting sinusoidal gratings are presented simultaneously to the two eyes for $4 \mathrm{sec}$. The stimulus contrast and spatiotemporal phase are systematically varied from trial to trial in the varied eye, whereas the grating configuration in the fixed eye does not change. $B$, Model of contrast gain control for binocular simple cells. The input to each eye is a drifting sinusoidal grating, with specified contrast $\left(C_{\mathrm{V}}\right.$ or $\left.C_{\mathrm{F}}\right)$ and phase $\left(\theta_{\mathrm{V}}\right.$ or $\left.\theta_{\mathrm{F}}\right)$. The monocular filter transforms the signal: the amplitude is multiplied by the monocular gain $\left(G_{\mathrm{V}}\right.$ or $\left.G_{\mathrm{F}}\right)$, and the monocular phase lag $\left(\varphi_{\mathrm{V}}\right.$ or $\left.\varphi_{\mathrm{F}}\right)$ is added to the signal phase. Signals from the two eyes are summed linearly and passed through a binocular filter, with specified binocular gain $\left(G_{\mathrm{B}}\right)$ and binocular phase $\operatorname{lag}\left(\varphi_{\mathrm{B}}\right)$. Finally, the signal is transformed by a static output nonlinearity. Some parameters in the model are contrast-dependent; these are marked by filled arrows. These parameters are allowed to vary freely as a function of contrast $\left(C_{\mathrm{V}}\right)$ in the varied eye. The model thus permits a distinction between contrast-dependent effects at a monocular site $\left(G_{\mathrm{V}}, \varphi_{\mathrm{V}}\right)$ or a binocular site $\left(G_{\mathrm{B}}, \varphi_{\mathrm{B}}\right)$. Potential effects of contrast at other sites $\left(G_{\mathrm{F}}, \varphi_{\mathrm{F}}\right.$, and the output nonlinearity) are considered in Results.

$L_{\mathrm{F}}$ (denoted $R_{\mathrm{V}}$ and $R_{\mathrm{F}}$, respectively) represent the linear contributions of the two eyes to the response.

If the form of the static output nonlinearity is fixed, the four parameters $S_{\mathrm{V}}, S_{\mathrm{F}}, L_{\mathrm{V}}$, and $L_{\mathrm{F}}$ suffice to predict the response of the system. The complex number $R_{\text {lin }}$, defined as the sum $S_{\mathrm{V}} L_{\mathrm{V}}+$ $S_{\mathrm{F}} L_{\mathrm{F}}$, is the linear response of the system: it is a quantity that specifies the phase and amplitude of the internal signal produced by the stimulus. The $F 1$ response, again a complex number, is given by $r=\xi\left(L_{\mathrm{V}} S_{\mathrm{V}}+L_{\mathrm{F}} S_{\mathrm{F}}\right)$, where $\xi$ is a function describing the effect of the static output nonlinearity on the amplitude and phase of the internal signal $R_{\text {lin }}$. The function $\xi$ alters the signal amplitude but leaves the phase fixed. The additional harmonics introduced by the output nonlinearity are ignored in our analysis.

In this study, we are interested in the effects of stimulus contrast on the linear filter coefficients $\left(L_{\mathrm{V}}\right.$ and $\left.L_{\mathrm{F}}\right)$ and possibly the output nonlinearity $(\xi)$. By determining the best-fitting linear filter coefficients $L_{\mathrm{V}}$ and $L_{\mathrm{F}}$ as a function of stimulus contrast in the varied eye, it is possible to determine whether an increase in contrast in one eye affects the contrast gain in that eye alone $\left(L_{\mathrm{V}}\right)$ or in both eyes $\left(L_{\mathrm{V}}, L_{\mathrm{F}}\right.$, and/or $\left.\xi\right)$. To emphasize the distinction between monocular and binocular effects, we will describe filter coefficients for each eye as the product of a monocular coefficient $\left(G_{\mathrm{V}} \mathrm{e}^{2 \pi \cdot \varphi_{\mathrm{V}}}\right.$ or $G_{\mathrm{F}} \mathrm{e}^{2 \pi \cdot \varphi_{\mathrm{F}}}$, respectively) and a common binocular component $\left(G_{B} \mathrm{e}^{2 \pi^{*} \varphi_{\mathrm{B}}}\right.$, for both eyes). This is shown in Figure $1 B$ and is described in greater detail below. This decomposition amounts to one method of parceling gain changes into monocular and binocular components and thus allows for a quantitative analysis of monocular versus binocular gain effects.
A final note concerns the form of the static output nonlinearity $\xi$. This is described according to the following formula (Heeger, 1992a, his Eq. 2):

$$
f(x)=\left\{\begin{array}{cl}
(x-T)^{\mathrm{n}} & \text { if } x-T \geq 0 \\
0 & \text { if } x-T<0
\end{array} .\right.
$$

Here, $x$ denotes the instantaneous magnitude of the input to the nonlinearity; $f(x)$ denotes the instantaneous response magnitude, $T$ denotes the response threshold, and $n$ denotes the nonlinearity's exponent. For most of this study, we let $n=2$ and $T=0$, which results in a half-squaring nonlinearity (Heeger, 1992a). The effect of the nonlinearity on a sinusoidal signal, as opposed to the instantaneous effect, can be determined from Equation 1 by analytic or numeric integration. For example, a half-squaring nonlinearity squares the signal amplitude and multiplies it by the constant $4 / 3 \pi$.

In sum, at fixed levels of stimulus contrast, the simple cell is modeled as a linear/nonlinear mechanism, with a linear filter followed by a static nonlinearity. Contrast-dependent changes in the parameters of this model are interpreted as the effects of monocular or binocular gain control mechanisms.

\section{Implementation of the basic model}

The experimental design is shown in Figure 1. A sinusoidal grating is presented to both the varied eye (V) and the fixed eye $(F)$. Each drifting grating is described by an amplitude parameter $\left(C_{\mathrm{V}}\right.$ or $\left.C_{\mathrm{F}}\right)$, which equals stimulus contrast, and a phase parameter $\left(\theta_{\mathrm{V}}\right.$ or $\left.\theta_{\mathrm{F}}\right)$. (The convention in this study is that the phase increases as the stimulus is shifted to the right along the time axis.) A linear filter in each eye converts the input into an internal signal. The amplitude of this signal equals the amplitude of the input $\left(C_{\mathrm{V}}\right.$ or $\left.C_{\mathrm{F}}\right)$ times the contrast gain of the corresponding filter $\left(G_{\mathrm{V}}\right.$ or $\left.G_{\mathrm{F}}\right)$. Likewise, the phase of the signal equals the phase of the input $\left(\theta_{\mathrm{V}}\right.$ or $\left.\theta_{\mathrm{F}}\right)$ plus the phase lag of the filter $\left(\varphi_{\mathrm{V}}\right.$ or $\left.\varphi_{\mathrm{F}}\right)$. The monocular signals are summed linearly, scaled by the binocular gain parameter $G_{\mathrm{B}}$, and shifted in phase by the binocular phase lag parameter $\varphi_{\mathrm{B}}$. Finally, the signal passes through a static output nonlinearity. Unless stated otherwise, the output nonlinearity is modeled as a half-squaring operation.

We now consider how the model parameters are determined from empirical data. To begin, consider the PSTHs of Figure 2, which displays the responses of a simple cell to dichoptically presented drifting sinusoidal gratings. Contrast is fixed at $50 \%$ in the right eye but is varied from 2.5 to $50 \%$ in the left eye (columns). Relative stimulus phase is also varied in steps of $45^{\circ}$ (rows). Each PSTH displays the averaged response of the neuron to a single stimulus cycle and therefore represents the average of 40 response cycles (i.e., five repetitions times eight stimulus cycles per repetition). Note that each PSTH response is quasi-sinusoidal and that the nonlinear distortion in the response is to a first approximation consistent with a half-squaring static output nonlinearity. Although only the $F 1$ responses are used to determine the model parameters, the model prediction (solid line curves) agrees reasonably well with the PSTH data overall.

Although systematic variations in the amplitude and phase of the $F 1$ response are observed in Figure 2 as a function of contrast and relative stimulus phase, these changes are difficult to interpret when presented in this format. We therefore use polar plots (Movshon et al., 1978; Ohzawa and Freeman, 1986) to summarize the $F 1$ responses of the neurons (Fig. 3). The phase and amplitude of the neural response are represented as phase and amplitude of the vectors shown in the plot. In the absence of an output 


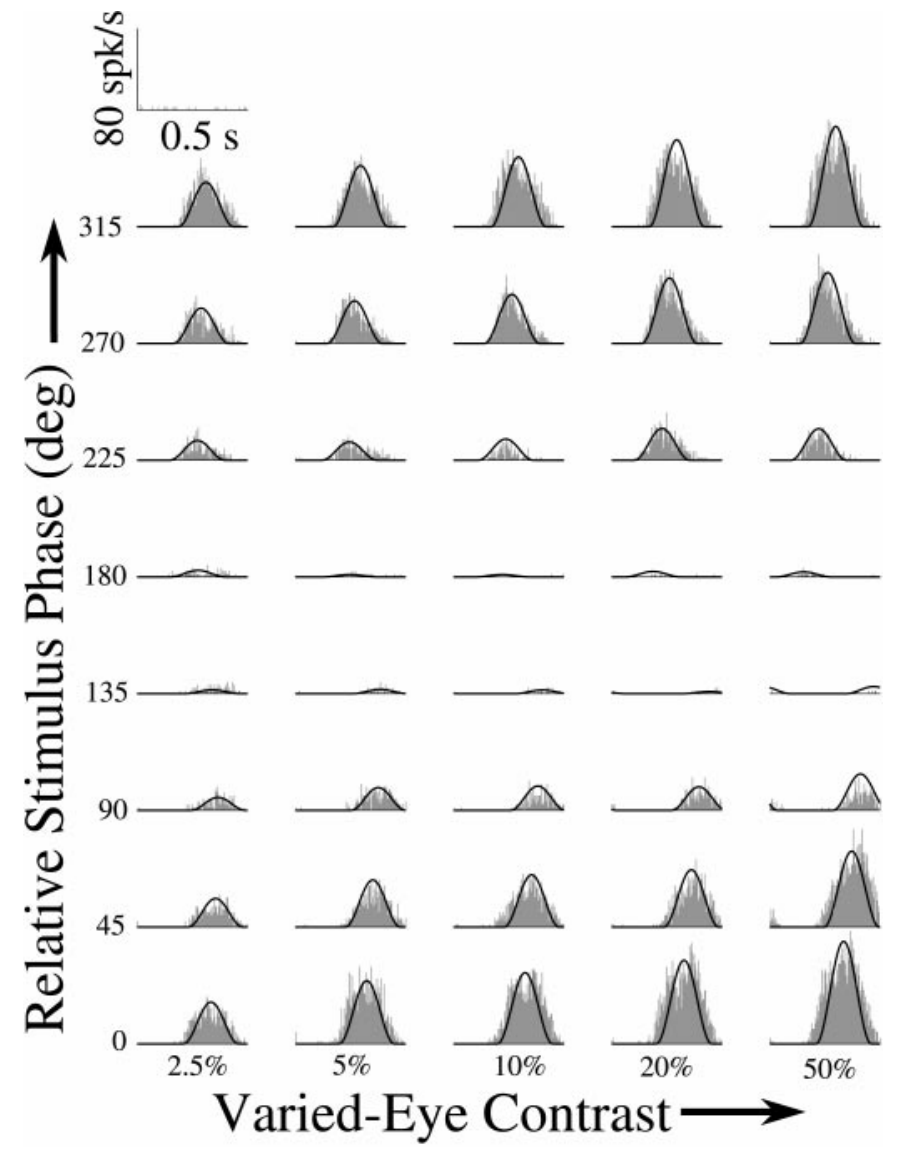

Figure 2. PSTHs for an experiment involving a binocular simple cell. Each PSTH represents the averaged response of the neuron to a full cycle of stimulation, for a given stimulus configuration. In one eye, contrast is fixed at $50 \%$. In the other, contrast is varied from 5 to $50 \%$ (columns) and stimulus phase is varied over a $360^{\circ}$ range (rows). These manipulations result in systematic changes in response amplitude and phase. The $F 1$ component of each PSTH was determined, and these values were used to extract model parameters. The fits of the model to the data are shown (solid-line curves). The PSTH in the top left-hand corner indicates the scale for this figure and displays the average response of the neuron when no stimulus was present.

nonlinearity, the neuron is linear, and its response $R_{\text {lin }}$ can be decomposed into varied-eye and fixed-eye components $\left(V_{\text {lin }}\right.$ and $\left.F_{\text {lin }}\right)$, which represent the contributions of the varied eye and fixed eye, respectively, to the response. Thus, $R_{\text {lin }}=V_{\text {lin }}+F_{\text {lin }}$. Figure $3 A$ illustrates how, as left-eye stimulus phase is varied over a $360^{\circ}$ range, the net response vector will follow a circular trajectory in the polar plot representation. By making a least square fit of a circle to this trajectory, one can determine the vector $V_{\text {lin }}$ as the radius of the circle, and the vector $F_{\text {lin }}$ as the offset of the circle from the origin. (Computationally, this is accomplished as follows. Regard $R_{\text {lin }}$ as a complex number, with amplitude and phase as shown in the polar plot. Then $R_{\text {lin }}$ may be viewed as a complexvalued function of the stimulus phase $\theta_{\mathrm{V}}$ of the varied eye. The complex numbers $V_{\text {lin }}$ and $F_{\text {lin }}$ can be determined as the $F 0$ and $F 1$ Fourier components, respectively, of this periodic function.)

Note that the circular response trajectory of an otherwise linear system (Fig. 3A, dashed line) is distorted by the system's output nonlinearity (solid line). If the form of the output nonlinearity is assumed known, one can recover the linear filter coefficients by inverse-transforming the data through the nonlinearity, then calculating $F 0$ and $F 1$ Fourier coefficients. If the output nonlinearity is modeled parametrically, one can instead use nonlinear regression methods. In this study, the filter parameters are determined by both methods: the inverse-transform method is used to provide the nonlinear regression algorithm with initial conditions for its search.

We now consider how binocular and monocular gain parameters are determined from estimates of $V_{\text {lin }}$ and $F_{\text {lin }}$. Consider, for example, an experiment with five contrast levels in the varied eye. We denote these as $C_{\mathrm{V}, 1}, \ldots, C_{\mathrm{V}, 5}$. Likewise, we use numerical subscripts of this sort for the contrast-dependent parameters of the model $\left(G_{\mathrm{F}}, G_{\mathrm{V}}, G_{\mathrm{B}}, \varphi_{\mathrm{V}}, \varphi_{\mathrm{F}}\right.$, and $\left.\varphi_{\mathrm{B}}\right)$. What effect does contrast in the varied eye have on the three gain parameters $G_{\mathrm{F}}$, $G_{\mathrm{V}}$, and $G_{\mathrm{B}}$ shown in Figure $1 B$ ? Hypothetically, gain control mechanisms could affect these parameters in three ways. First, as contrast is raised in the varied eye, the corresponding monocular gain parameter $G_{\mathrm{V}}$ could be reduced. This will minimize the effect of the contrast change on the responses of binocular simple cells. Alternatively, the binocular gain $\left(G_{\mathrm{B}}\right)$ or the fixed-eye gain $\left(G_{\mathrm{F}}\right)$ could be reduced, leading in either case to suppression of the signal from the fixed eye. However, only two gain parameters can actually be determined from the data in this study: these are the net gain parameters $G_{\mathrm{V}} G_{\mathrm{B}}$ and $G_{\mathrm{F}} G_{\mathrm{B}}$ for the two eyes. In particular, a change in $G_{\mathrm{B}}$ cannot be disambiguated from a concurrent change in $G_{\mathrm{V}}$ and $G_{\mathrm{F}}$. To resolve this problem, we will assume that $G_{\mathrm{F}}$, the fixed-eye gain, remains fixed throughout a series of trials. In other words, we assume that an increase in the varied eye's contrast does not affect the monocular filter associated with the fixed eye.

The effects of contrast variations are quantified as follows. First, because signal-to-noise levels are usually greatest at high contrasts, the high-contrast condition $\left(C_{\mathrm{V}, 5}\right)$ is used as a reference against which the other conditions are compared. For the highcontrast condition, estimates are made of the two net gain parameters $\left(G_{\mathrm{V}, 5} G_{\mathrm{B}, 5}\right.$ and $\left.G_{\mathrm{F}, 5} G_{\mathrm{B}, 5}\right)$ and the two net phase lag parameters $\left(\theta_{\mathrm{V}, 5}+\theta_{\mathrm{B}, 5}\right.$ and $\left.\theta_{\mathrm{F}, 5}+\theta_{\mathrm{B}, 5}\right)$. Similar estimates are made for the other contrast conditions, $C_{1}, C_{2}, C_{3}$, and $C_{4}$. However, these are reported as relative gain parameters. For example, the relative binocular gain for contrast condition 1 is the ratio $G_{B, 1} / G_{B, 5}$, which is calculated from the net gain parameters as follows:

$$
\frac{G_{\mathrm{B}, 1}}{G_{\mathrm{B}, 5}}=\frac{G_{\mathrm{F}, 1} G_{\mathrm{B}, 1}}{G_{\mathrm{F}, 5} G_{\mathrm{B}, 5}} .
$$

(Note that this equation works because $G_{\mathrm{F}, 1}=G_{\mathrm{F}, 5}$.) Likewise, the relative monocular gain $\mathrm{G}_{\mathrm{V}, 1} / \mathrm{G}_{\mathrm{V}, 5}$ is calculated from the net gain parameters as follows:

$$
\frac{G_{\mathrm{V}, 1}}{G_{\mathrm{V}, 5}}=\frac{G_{\mathrm{V}, 1} G_{\mathrm{B}, 1}}{G_{\mathrm{V}, 5} G_{\mathrm{B}, 5}} \cdot \frac{G_{\mathrm{F}, 5} G_{\mathrm{B}, 5}}{G_{\mathrm{F}, 1} G_{\mathrm{B}, 1}} .
$$

The relative binocular phase $\operatorname{lag} \theta_{\mathrm{B}, 1}-\theta_{\mathrm{B}, 5}$ and the relative monocular phase lag $\theta_{\mathrm{V}, 1}-\theta_{\mathrm{V}, 5}$ are calculated in a similar way from the net phase lag parameters for these conditions, using addition and subtraction rather than multiplication and division.

In sum, we quantify the effect of contrast on the relative value of contrast gain at both a monocular site $\left(G_{\mathrm{V}}\right)$ and a binocular site $\left(G_{\mathrm{B}}\right)$. This assumes that contrast in the varied eye does not affect other aspects of the simple cell model, such as fixed-eye gain $\left(G_{\mathrm{F}}\right)$ and the output nonlinearity. We return to this assumption later, where we show that it is not too critical for interpreting the results. 

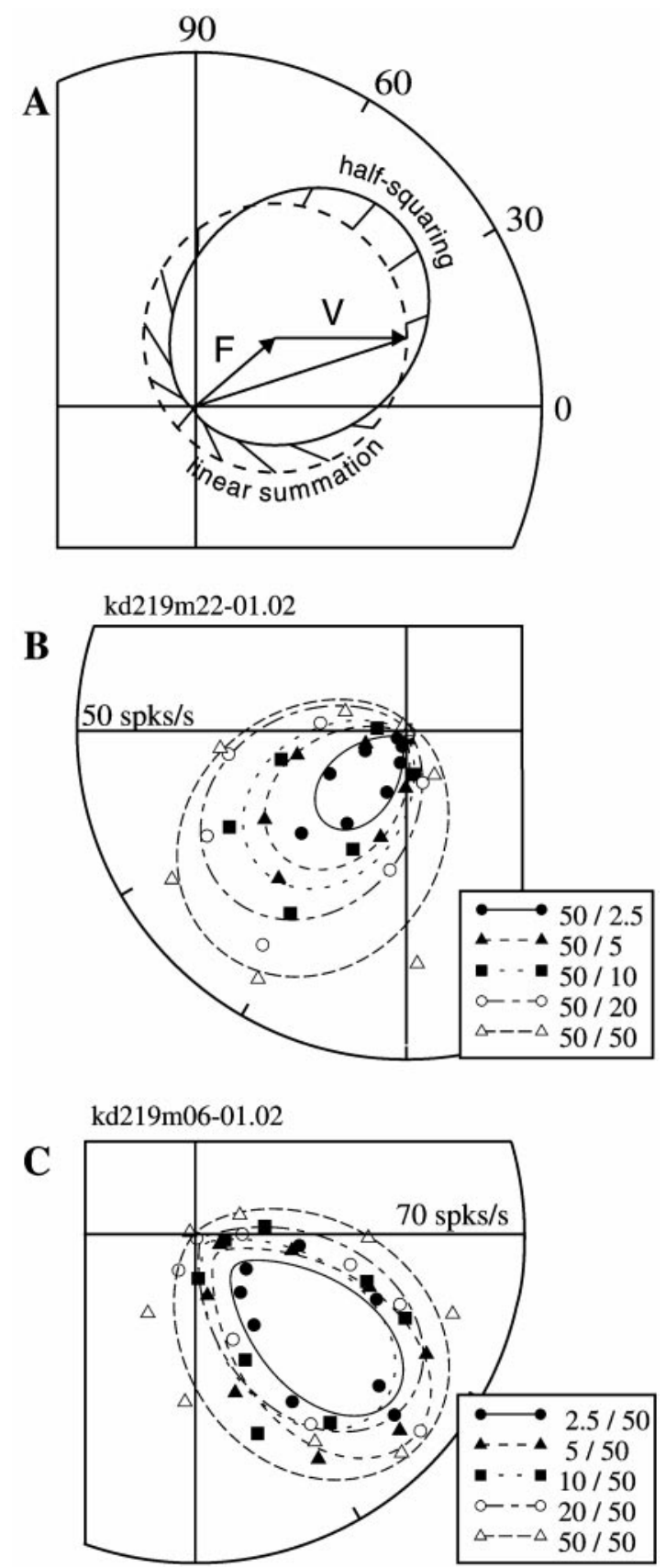

Figure 3. A, This polar plot illustrates the amplitude and phase of the $F 1$ response of a hypothetical neuron stimulated dichoptically by drifting sinusoidal gratings. If the neuron is strictly linear, then the neural response equals the vectorial sum of a varied-eye $(V)$ and a fixed-eye $(F)$ response component. As the phase of the stimulus of the varied eye is varied over a $360^{\circ}$ range, the phase of $V$ varies by equal amounts. This causes the net neural response to follow a circular trajectory (dashed line). Fourier analysis of this trajectory provides a direct estimate of the magnitude and phase of $V$ and $F$. If this analysis is repeated for multiple levels of contrast in the varied eye, then one can determine the relative contributions of monocular gain control (which affects only $V$ ) and of binocular gain control (which affects both $V$ and $F$ ). This analysis must incorporate the output nonlinearity, which distorts and generally elongates the response trajectory (solid line). $B, C$, These polar plots show the $F 1$ responses of two simple cells to dichoptically presented sinusoidal gratings. The polar plot in $B$ corresponds to the PSTH data of Figure 2. In $B$ and $C$, contrast was varied from 2.5 to $50 \%$ in one eye but was fixed at $50 \%$ in the other eye. Each contrast level is represented by a unique symbol and a unique line style, and the corresponding left-eye and right-eye contrast values are shown in the legend. At each contrast level,

\section{Examples of gain control effects}

Figure $3 B$ illustrates the $F 1$ response data for the neuron shown in Figure 2. For this cell, contrast was fixed at $50 \%$ in the left eye and varied from 2.5 to $50 \%$ in the right eye. Each contrast level is represented by a unique symbol and line style: the eight instances of a given symbol correspond to measurements made at different relative stimulus phase values, whereas each curve represents the model fit to the phase-versus-response function. Note that at all contrasts, the phase-versus-response trajectory is quasi-elliptical, reflecting the distortions introduced by the output nonlinearity. Each ellipse has two basic features. The first is the approximate center of the ellipse. This reflects the contribution of the fixed eye to the response. The second is the approximate radius of the ellipse. This reflects the part of the neural response that depends on the stimulus phase in the varied eye.

Three observations can be made from Figure $3 B$. First, none of the ellipses are centered at the origin of the polar plot. This indicates that the fixed eye makes a contribution to the response at all levels of contrast $(2.5-50 \%)$ in the varied eye. Second, the size of the ellipses increases somewhat as a function of contrast in the varied eye. This is to be expected if an increase in contrast in one eye increases the effective signal strength from that eye. Third, although contrast in the varied eye increases by a factor of 20, the approximate radius of the ellipse increases by a much smaller amount. In a completely linear system, the radius would be proportional to the stimulus contrast for the varied eye. However, the magnitude of the increase is difficult to gauge by eye, in particular because of the distortions associated with the output nonlinearity. Data fitting indicates that the signal from the varied eye increases by a factor of only 2.4 as contrast is raised from 2.5 to $50 \%$. This indicates the presence of an effective gain control mechanism that maintains a relatively constant signal level despite large variations in input strength.

A second example is shown in Figure $3 C$. The same three features are evident here. At all contrast levels, the fixed eye makes a contribution to the response. As contrast is increased in the varied eye, a contrast-dependent increase in signal strength is reflected in an increased radius of the elliptical trajectory. Again, however, the change in radius is much smaller than the 20 -fold change in the input strength.

In Figure 4, the responses of the cells from Figure 3, $B$ and $C$, are replotted, but this time the phase and amplitude components of the responses are shown separately. As stimulus phase in the varied eye is changed, the relative phase of the stimulus of one eye relative to the others is systematically varied. Thus, the curves in Figure 4, $A$ and $B$, are relative-phase tuning curves: they show the relation between relative stimulus phase in the two eyes and response amplitude. Similar curves have been described elsewhere (Ohzawa and Freeman, 1986, 1994; Smith et al., 1997a) and are of interest because they demonstrate the sensitivity of simple cells to variations in relative stimulus phase between the two eyes.

\section{$\leftarrow$}

the relative phase of the left-eye and right-eye gratings was also varied, so that eight data points were collected for each of five contrast levels. Symbols denote individual data points, whereas lines represent the model fit to the data. The approximate radius of each curve represents the contribution made by the varied eye to the response of the cell. For each cell, the radius grows as a function of varied-eye contrast, but the rate of growth is small when compared with the 20 -fold change in contrast. This indicates that the increase in varied-eye contrast reduces the response gain of the monocular and/or binocular pathways. 
Figure 4. Amplitude $(A, B)$ and phase $(C, D)$ of responses of binocular simple cells to drifting gratings. Data in $A$ and $C$ are replotted from Figure $3 B$, whereas data in $B$ and $D$ are replotted from Figure $3 C$. Because stimulus phase in the fixed eye is constant, the relative interocular phase is determined by the stimulus phase in the varied eye. At each contrast level in the varied eye, changes in the relative interocular phase produce systematic changes in response amplitude (Fig. $5 A, B$ ) and response phase (Fig. $5 C, D$ ). Consistent with linear binocular interactions in the simple cell, the relative-phase tuning functions in Figure 5, $A$ and $B$, are unimodal, with a peak and a trough reflecting the periodic summation and cancellation of the inputs from the two eyes (Ohzawa and Freeman, 1986). The fits of the model to the data are shown by the solid-line curves. The apparent $360^{\circ}$ discontinuities in the phase-versus-phase plots $(C, D)$ reflect the fact that response phase is a cyclic quantity.
A

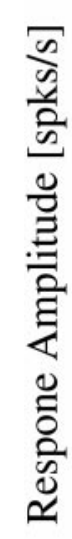

$\mathbf{C}$

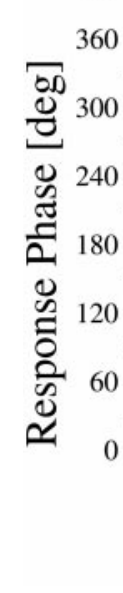

\section{$\mathrm{kd} 219 \mathrm{~m} 22-01.02$}

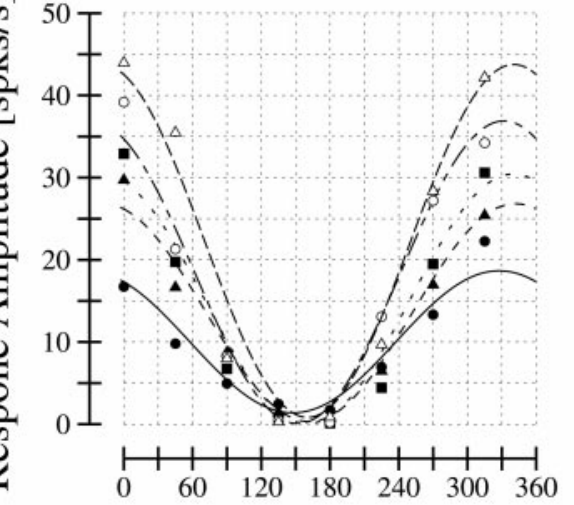

\section{kd219m22-01.02}

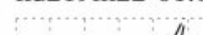

B

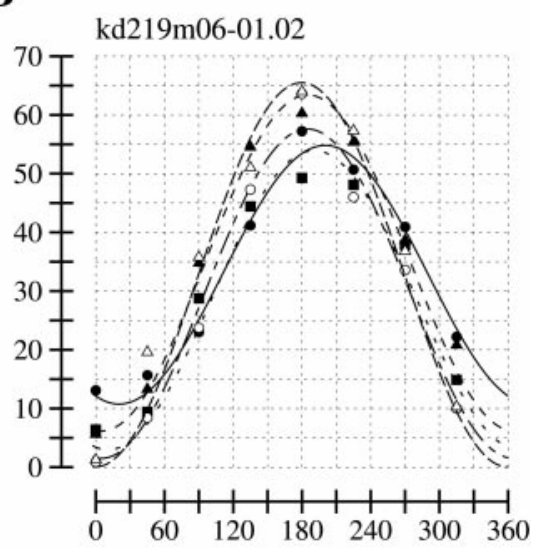

D

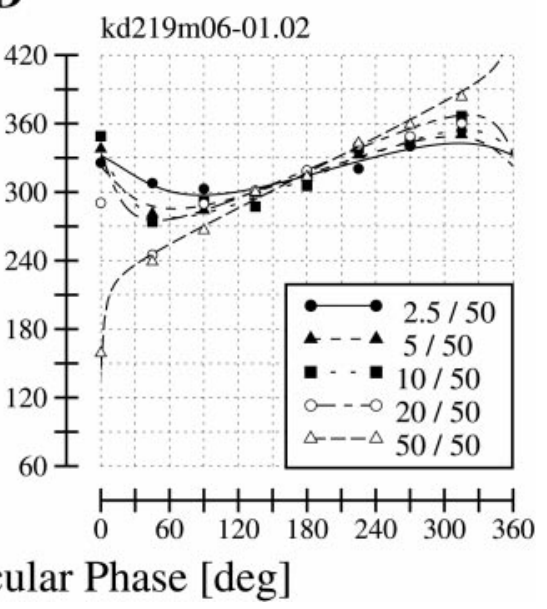

Figure 4, $C$ and $D$, shows the effect of varied-eye phase on the response phase. The apparent discontinuities in the graph reflect the fact that response phase is a cyclic quantity. The quantitative model produces reasonable fits to the data in both the amplitude and phase domains (solid lines).

As contrast is increased in the varied eye, marked reductions in response gain are observed. This is reflected in changes in the best-fitting monocular and binocular gain parameters of the quantitative model. Figure 5, $A$ and $B$, shows the effect of contrast on monocular gain for the two neurons (solid lines). Each data point is normalized relative to the monocular gain at $50 \%$ contrast. Thus, these graphs show the effect of contrast on relative monocular gain, which necessarily equals 1 at $50 \%$ contrast. For both neurons, increases in contrast lead to sharp reductions in monocular gain. This demonstrates that increasing contrast in one eye leads to a large reduction in response gain, which is associated with the signal from that eye only. Relative binocular gain shows almost no change for one cell (Fig. 5C) and little change for the other cell (Fig. 5D). This indicates that an increase in contrast in one eye has little effect on the effective strength of the signal from the other eye.

These changes in gain are described in terms of the monocular gain slope and binocular gain slope. These values equal the slope of the logarithmic contrast versus gain plots. A gain slope of -1 indicates that response gain is inversely proportional to contrast, and thus signifies gain control that is $100 \%$ effective; i.e., the output signal level remains constant despite any changes in stimulus contrast. On the other hand, a slope of 0 denotes the absence of gain control. For the cell described in Figure 5, $A$ and $C$, the monocular and binocular gain slopes equal $-0.75 \pm 0.01$ and $0.00 \pm 0.01$, respectively, whereas for the cell described in Figure $5, B$ and $D$, they are $-0.67 \pm 0.03$ and $-0.11 \pm 0.02$. (SE estimates for these values were determined based on MonteCarlo simulations, in which random data sets were generated by uniform random sampling of the multiple response cycles associated with each trial.) Thus, these cells exhibit similar, substantial reductions in monocular gain, whereas the binocular gain is quite flat.

\section{Population data}

Figure 6 summarizes the effect of contrast on relative gain and relative phase lag, in those cases in which contrast was fixed at $48-51 \%$ in one eye. Extrapolation or interpolation is used in this and subsequent population figures to normalize all data relative to $50 \%$ contrast (Fig. $6 A$ ) and to produce averages and SDs for selected contrast values (Fig. $6 B$ ). For all neurons, relative monocular gain (Fig. 6A) drops off steeply with increasing contrast, but never quite enough to completely compensate for the effects of increased contrast (dotted line). The average monocular gain at $5 \%$, relative to the gain at $50 \%$, is $5.0(\mathrm{SD}=1.9)$. This indicates that as contrast changes by a factor of 10 (i.e., from 5 to $50 \%$ ), monocular gain is reduced by a factor of 5 on average.

For most cells, binocular gain is also reduced as a function of stimulus contrast, although these changes are considerably smaller than the monocular gain reductions. The average relative binocular gain at $5 \%$ contrast is $1.2(\mathrm{SD}=0.3)$, indicating a 

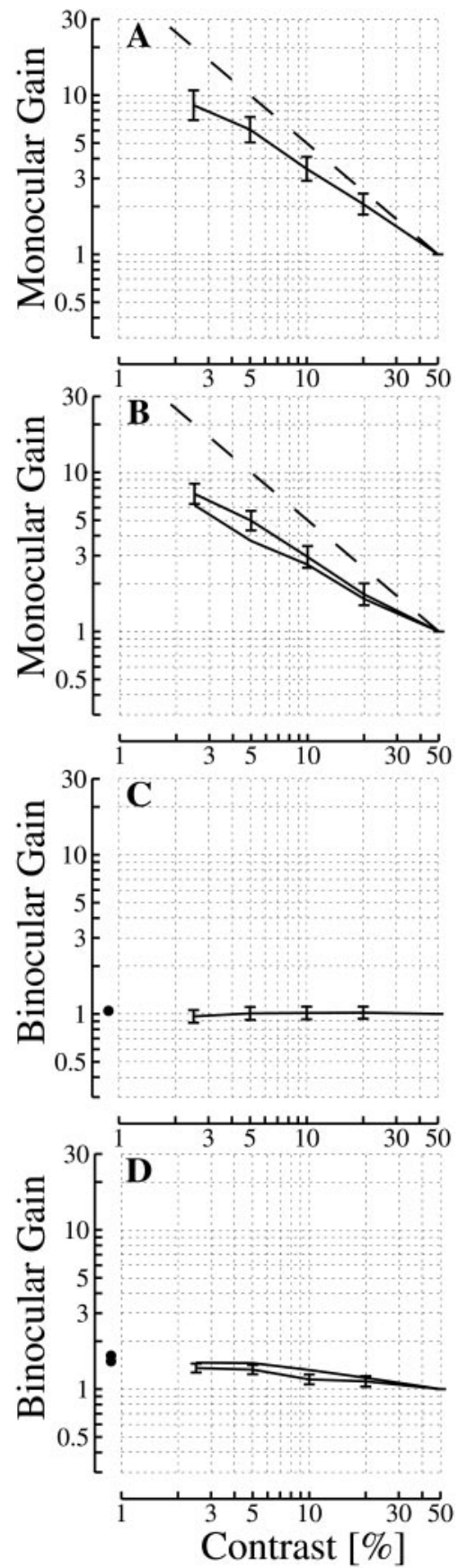

Figure 5. The effect of varied-eye contrast on monocular and binocular gain parameters for the two cells of Figures 3 and 4 . All parameters values are normalized to the corresponding value in the $50 \%$ contrast condition. Data from the cell of Figure $3 B$ are shown in $A$ and $C$, whereas data from the cell of Figure $3 C$ are shown in $B$ and $D$. Error bars denote the parameters' formal SE values, as determined by the LevenbergMarquardt procedure, and in $B$ and $D$, solid lines without error bars denote the results obtained from a repetition of the experimental procedure for this cell. As contrast in the varied eye increases, the monocular gain drops dramatically for both neurons, whereas the binocular gain shows either no change $(C)$ or a modest reduction $(D)$. For comparison, note that if a monocular gain control system were $100 \%$ effective at counteracting the effects of increased contrast in one eye, then a slope of -1 would be obtained on these $\log -\log$ axes $(A, B$, dashed lines $)$. The filled circles in the margins of $C$ and $D$ indicate the relative binocular gain in the absence of stimulation in the varied eye $(0 \%$ contrast). weakly significant effect of binocular gain control $(t=2.4, p<$ 0.05 , two-sided $t$ test). Because binocular gain is reduced only by a factor of 1.2 as the contrast is changed by a factor of 10 , it is clear that the binocular gain control system plays a relatively minor role.

The blocks of data include monocular control trials in which the fixed eye contrast is $\sim 50 \%$, whereas a blank screen with no contrast is presented to the varied eye. These data are analyzed with a separate computation that does not contribute to the data fitting overall. From the phase and amplitude of the responses, we calculate gain $\left(G_{\mathrm{F}} G_{\mathrm{B}}\right)$ and phase lag $\left(\varphi_{\mathrm{F}}+\varphi_{\mathrm{B}}\right)$ of the fixed eye's linear filter, in the absence of input from the varied eye. From these values, we determine the relative binocular gain and phase lag for the $0 \%$ varied-eye contrast condition, in the same way that these values are computed for all other contrast conditions (Eq. 2). The relative binocular gain values are shown in Figure $6, C$ and $D$ (filled circles). These indicate the total effect that a $50 \%$ grating in the varied eye has on the transmission of a signal from a $50 \%$ grating in the fixed eye. The average relative binocular gain at $0 \%$ contrast is $1.2(\mathrm{SD}=0.53)$, a value that is not statistically distinct from $1.0(t=1.65, p>0.1)$. We conclude that simultaneous presentation of the $50 \%$ contrast grating to one eye has relatively little effect on the signal from the $50 \%$ grating in the other eye.

Figure $6, E$ and $F$, shows the effect of contrast on monocular phase lag. The average phase lag at $5 \%$, relative to the phase lag at $50 \%$, is not statistically different from $0^{\circ}$ (mean $=-0.9^{\circ}, \mathrm{SD}=$ $13.4^{\circ}, t=0.3, p>0.1$, two-sided $t$ test).

The finding of modest net changes in the phase lag parameter is consistent with the observation of Smith et al. (1997a) that for dichoptic sinusoidal stimuli, a change in contrast in one eye has little effect on the optimal phase of the relative-phase tuning function. On the other hand, for V1 simple cells stimulated monocularly with $2 \mathrm{~Hz}$ sinusoidal gratings, Dean and Tolhurst (1986) report a mean phase advance of $33.2^{\circ}$ between 5 and $25 \%$ contrast, although the SD is large $\left(25.3^{\circ}\right)$. Similar results have been reported for macaque V1 simple cells (Carandini et al., 1993, 1997). This raises the possibility that the phase advances seen with monocular stimulation are actually mediated at a binocular site and that in the present study the underlying mechanism is already saturated by the $50 \%$ contrast in the fixed eye. However, direct comparisons of the various studies are made difficult by the low statistical reliability of phase measurements made at low contrasts.

Changes in the binocular phase parameter are consistently minimal (Fig. 6G,H). The average binocular phase lag at 5\% contrast, relative to the phase lag at $50 \%$ contrast, is $3.7^{\circ}(\mathrm{SD}=$ $\left.11.2^{\circ}\right)$. This effect is not statistically significant $(t=1.6, p>0.1$, two-sided $t$ test).

In sum, as contrast is raised from 5 to $50 \%$, the monocular gain drops by a factor of 5 on average, whereas much more limited changes are seen for binocular gain and binocular phase lag.

\section{Low reference contrast}

In the data described so far, the contrast for the fixed eye is high $(50 \%)$. To establish the generality of the results, we conducted some experiments with a low reference contrast in the fixed eye.

Figure 7 illustrates the results from a block of trials in which contrast is fixed at $10 \%$ in the left eye and varied from 4 to $50 \%$ 
Figure 6. Gain parameters as a function of varied-eye contrast for experiments with a high $(\sim 50 \%)$ contrast in the fixed eye. Each line in $A, C, E$, and $G$ represents the outcome from relative to the corresponding parameter value at $50 \%$ contrast. (In some cases, this normalization required a slight interpolation or extrapolation to the $50 \%$ value, attributable to contrast correction.) Point-by-point averages of these curves are shown in $B, D, F$, and $H$, respectively, with $\pm 1 \mathrm{SD}$ error bars at five arbitrarily chosen contrast levels. Note that there is a difference in scale between the phase data from individual experiments $(E, G)$ and the averaged phase data $(F, H) . A, B$, For all experiments, the monocular gain parameter is sharply reduced by stimulus contrast: the monocular gain slope, or slope of the contrast-versus-gain curve, is nearly -1 (dotted line) in every case. $C, D$, Most cells show contrast-dependent reductions in binocular gain, but these effects are modest in comparison to the monocular gain effects. The filled circles in the margins indicate the relative binocular gain parameter with $0 \%$ contrast in the varied eye. $E, F$, The effect of increased contrast on monocular phase lag is somewhat variable, at least in part because estimates of this parameter were noisy. On average, no net change in this parameter is seen as contrast is raised from 5 to $50 \%$ in the varied eye. $G, H$, An increase in contrast from 5 to $50 \%$ has at best a modest effect on the binocular phase lag. On average, a contrast-induced binocular phase lag is observed when the 0 and $50 \%$ contrast conditions are compared, although a single experiment. All parameter values are normalized this effect is highly variable.
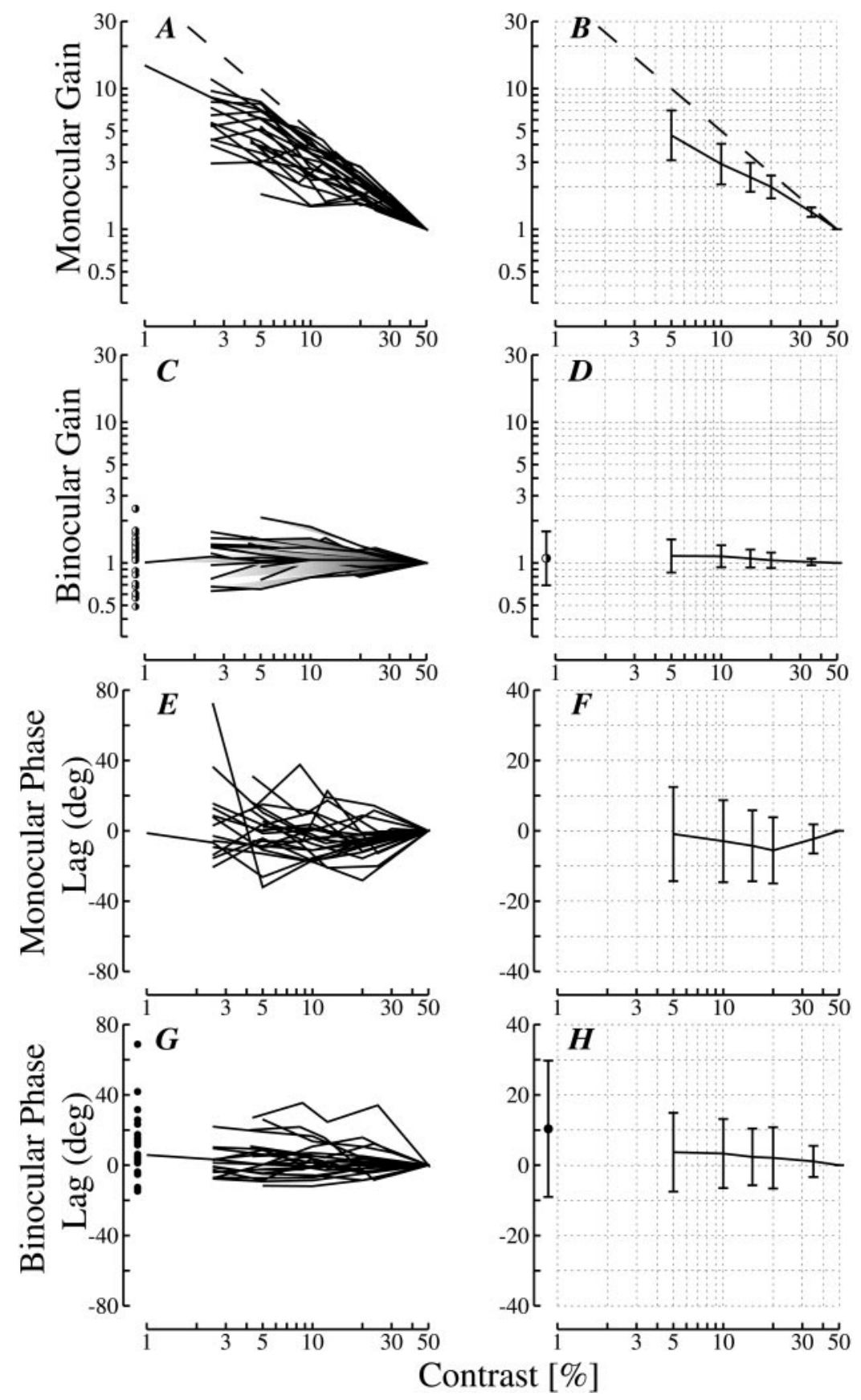

in the right eye. As in the case of the high-contrast examples above, the monocular gain drops rapidly with contrast (Fig. 7C). The binocular gain is nearly flat (Fig. 7D), and the binocular phase lag shows little change with contrast (data not shown). A considerable monocular phase advance is evident as contrast is raised from 4 to $8 \%$ (Fig. $7 B$ ), although the standard error of measurement is high.

Figure 8 summarizes contrast-dependent changes in gain parameters for blocks of trials in which a low contrast $(4-10 \%)$ is present in the fixed eye $(n=13)$. Strong reductions in monocular gain are evident (Fig. $8 A, B$ ), whereas the effects of contrast on binocular gain are weak (Fig. $8 C, D$ ). At $5 \%$ contrast, the average relative monocular gain is $3.6(\mathrm{SD}=2.0)$, whereas the average relative binocular gain is $1.3(\mathrm{SD}=0.79)$. Thus, as contrast is raised from 5 to $50 \%$, monocular gain is reduced on average by a factor of 3.6, whereas binocular gain drops by a factor of 1.3 on average. As shown in Figure $8 C$ (filled circles), the average relative binocular gain at $0 \%$ contrast is $1.1(\mathrm{SD}=0.5)$. This value is not statistically different from a ratio of $1(t=0.58, p>$ 0.1 , two-sided $t$ test). Thus, even if stimulus contrast in one eye (the fixed eye) is low, the presentation of a $50 \%$ grating to the other eye does not consistently suppress the signal generated by the fixed eye.

Note that there is an outlier in this data set (Fig. 8A,C). Although this cell passed our signal-to-noise criterion, input from one of the two eyes was predominately suppressive, and the cell exhibited anomalous relative-phase tuning functions. The model parameters are not statistically well constrained for this neuron, presumably because of the absence of sufficient linear driving input from one of the eyes. 


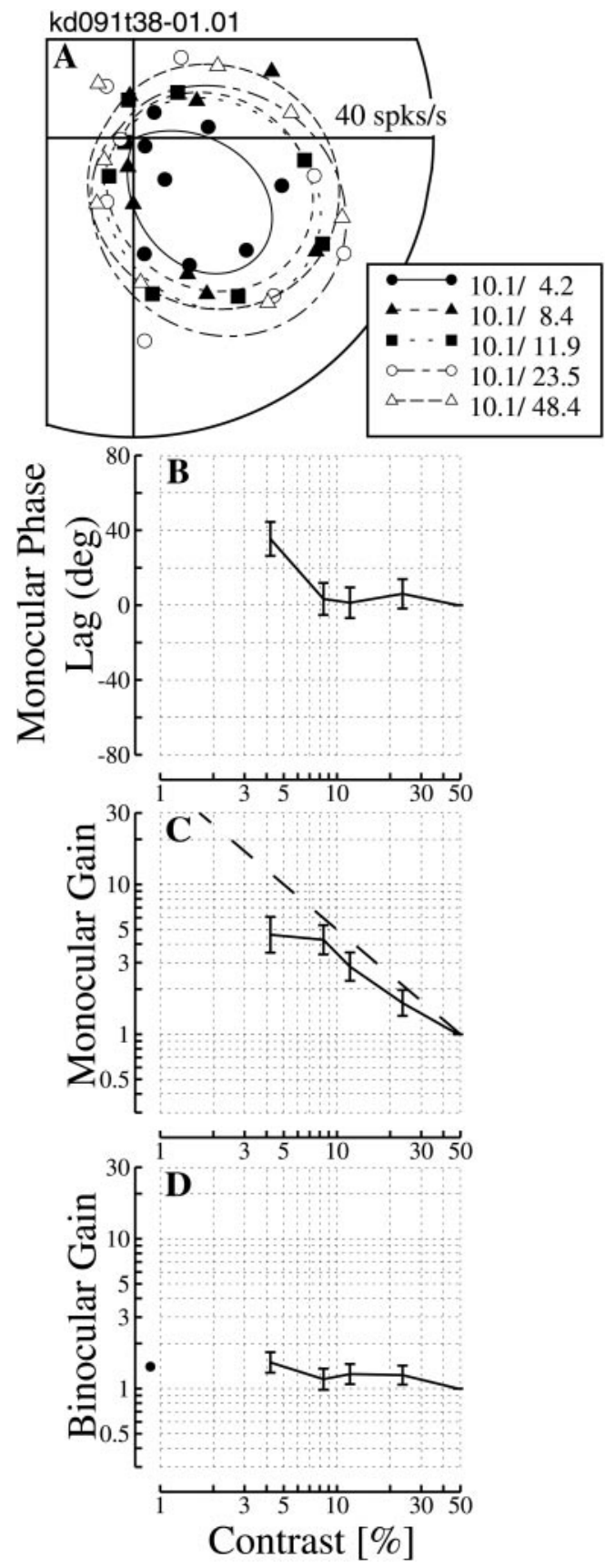

Figure 7. Analysis of a binocular simple cell stimulated with relatively low contrast $(10 \%)$ in the fixed eye. Data are presented in the format of Figures 3 and 5. $A$, Polar plot representation of the responses. $B$, A noisy signal at low contrasts may be responsible for this sharp effect of variedeye contrast on the estimated monocular phase parameter. $C, D$, As for the experiments with $50 \%$ contrast in the fixed eye, an increase of contrast in one eye sharply reduces the monocular gain but has little or no effect on the binocular gain.

\section{Monocular and binocular gain slopes}

The slopes of the contrast-versus-gain curves on log-log axes were used to assess the effectiveness of contrast gain control as varied-eye contrast was changed from 5 to $50 \%$. As mentioned above, a contrast gain slope of -1 indicates that the gain control system is $100 \%$ effective, whereas a slope of 0 indicates that the system is completely ineffective. Figure 9 summarizes the mon-
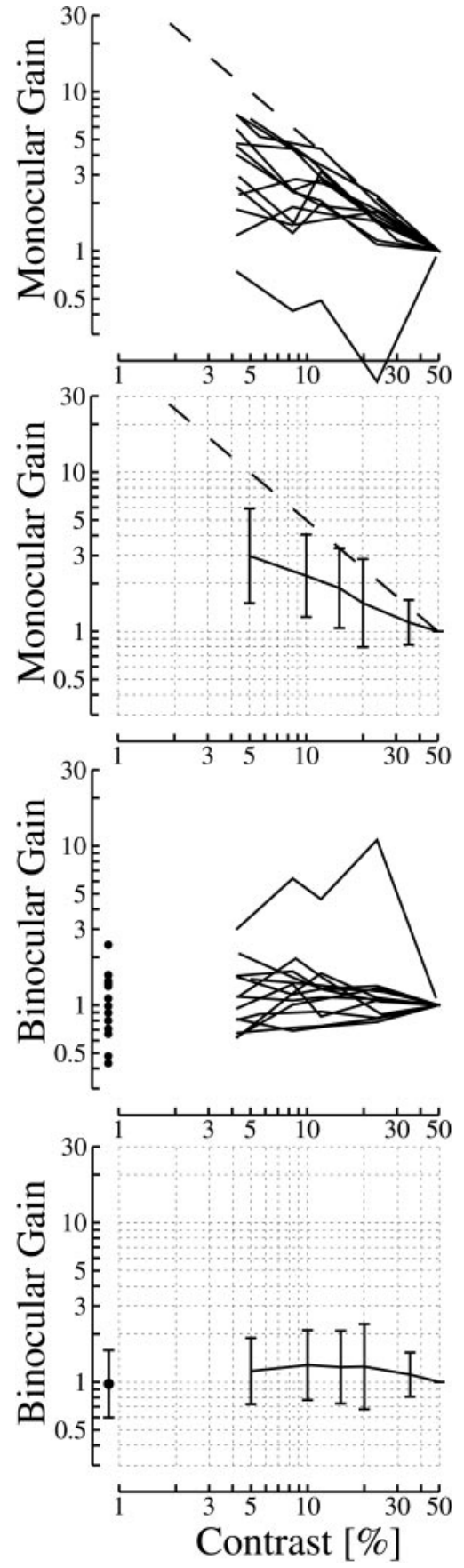

Figure 8. Results from experiments with low contrast (4-10\%) in the fixed eye are shown here, following the format of Figure 6 . As in the case of the fixed high-contrast experiments, an increase in varied-eye contrast has weak and inconsistent effects on the binocular gain $(C, D)$ but sharply reduces the monocular gain $(A, B)$. Phase data are not shown because of the difficulty of obtaining reliable phase estimates under low-contrast conditions.

ocular gain slopes for the high (filled circles) and low (unfilled circles) contrast conditions. For the high-contrast conditions, the monocular gain slope is always negative, with values ranging from -0.85 to -0.27 , and a mean value of $-0.64(\mathrm{SD}=0.15)$. The binocular gain slope ranges from -0.33 to 0.16 , with a mean value of $-0.05(\mathrm{SD}=0.11)$. Although the binocular gain slope is $<0$ on average ( $t=2.4, p<0.05)$, it is positive in 7 of 23 cases, indicating that reductions in binocular gain do not always occur as contrast 
$\mathbf{A}$

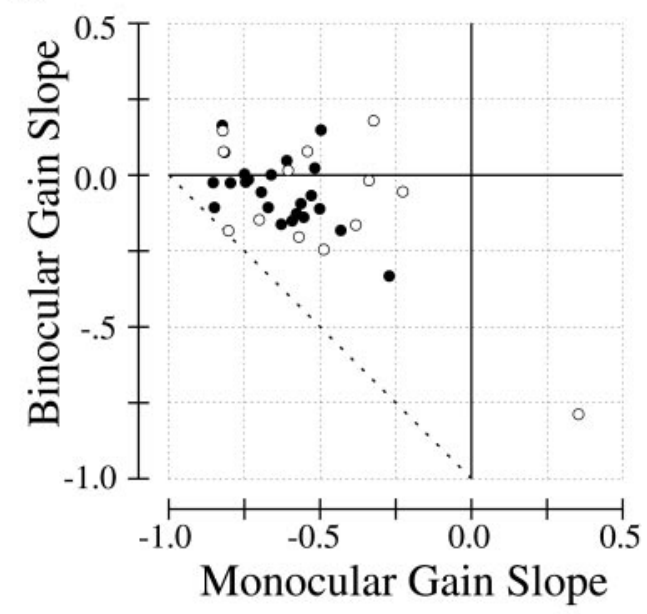

B

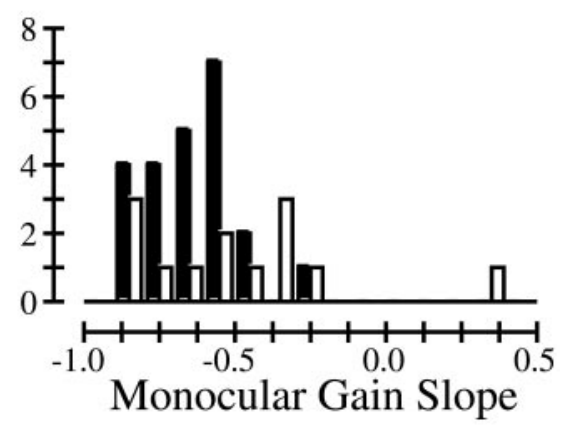

C

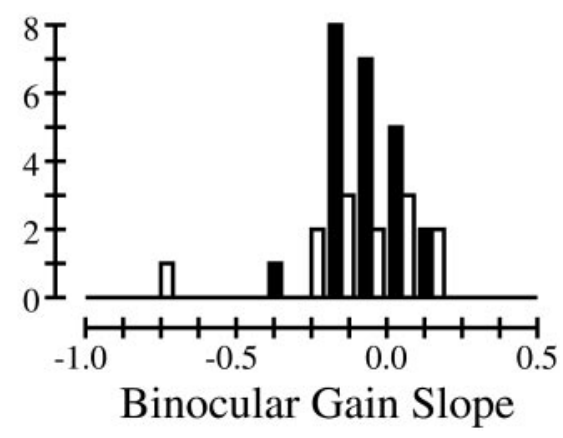

Figure 9. Monocular and binocular gain slopes are shown for experiments with a high contrast $(50 \%$; filled symbols) or a lower contrast (4-10\%; unfilled symbols) in the fixed eye. These slopes are calculated from the logarithmic plots of contrast versus gain. $A$, Monocular gain slopes are plotted against binocular gain slopes. When the sum of the monocular and binocular gain slope equals $-1,100 \%$ gain control has been achieved; this is denoted by the dotted line. B, Apart from an extreme outlier, the monocular gain slopes are consistently negative, with a mean value of $-0.48(\mathrm{SD}=0.32)$ and $-0.64(\mathrm{SD}=0.15)$ for the low- and high-contrast experiments, respectively. Thus, contrast-dependent gain reductions at a monocular site play a major role in determining the responses of binocular simple cells. These monocular gain slopes values can be compared to a value of -1 , which corresponds to $100 \%$ effective gain control. $C$, Binocular gain slopes cluster near 0 , indicating a less important role for binocular gain reductions.

increases. For the low-contrast experiments, the mean monocular and binocular gain slopes are $-0.48(\mathrm{SD}=0.32)$ and $-0.10(\mathrm{SD}$ $=0.25$ ), respectively. Thus, for these experiments too, the monocular gain control mechanism makes the major contribution.

\section{Relation to ocular dominance}

In this study, we have examined how the responses of individual neurons are affected by variations in the interocular contrast ratio. One could just as well ask how unequal contrasts affect the distribution of activity among the ocular dominance channels. This is of interest, for example, if the ocular dominance channel organization plays a role in binocular contrast summation (Anderson and Movshon, 1989). We have therefore examined whether our results depend on the ocular dominance of the neurons under investigation.

For each neuron, we calculated a varied-eye dominance value $(O D I)$ that was based on monocular responses to high-contrast stimuli ( $\sim 50 \%$ contrast). The ODI is calculated by the formula

$$
O D I=\frac{V}{F+V},
$$

where $V$ is the response rate (spikes per second) to varied-eye stimulation, and $F$ is the response to fixed-eye stimulation (see Macy et al., 1982). ODI equals 0 for neurons dominated by the fixed eye, 1 for neurons dominated by the varied eye, and 0.5 for binocularly balanced neurons. Monocular response data were obtained either from monocular spatial frequency tuning trials or, if available, from monocular control trials randomly interleaved with the main dichoptic trials.

Figure 10 shows the relationship between $O D I$ and the gain slope values. The correlation between $O D I$ and monocular gain slope is not significant for either the high-contrast $(r=-0.05, p>$ $0.1)$ or low-contrast $(r=0.28, p>0.1)$ conditions, although most of the lowest values of monocular gain slope are associated with binocularly balanced neurons (ODI near 0.5$)$, nor is a significant relationship found between $O D I$ and the binocular gain slope $(r=$ $0.23, p>0.1$, high contrast; $r=0.25, p>0.1$, low contrast) or between ODI and the sum of the monocular and binocular gain slopes $(r=0.14, p>0.1$, high contrast; $r=0.40, p>0.1$, low contrast). In effect, the operation of the gain control mechanisms is not strongly dependent on the ocular dominance index of the neuron under investigation.

\section{Control procedures}

Our main result is that monocular gain, but not binocular gain, is strongly reduced as stimulus contrast is increased. To help ensure that this conclusion is not sensitive to specific choices made in the construction of the model, we examined some variants of the gain control model of Figure $1 B$. Table 1 summarizes the results, and two examples are shown in Figures 11 and 12. The quality of the model fits to the data are evaluated in terms of percentage residual error, which ranges from $0 \%$ (a perfect fit) to $100 \%$ (the worst fit). This is the percentage of the variance of the data on the polar plots accounted for by the model prediction. We do not, however, use the F-test to calculate probability scores for the various models. Thus, this is essentially a heuristic approach to evaluating the models and should not be viewed as a replacement for the basic conclusions presented above.

Consider three basic models. The standard model, described in previous sections, allows the net gain and phase lag of each eye to change with stimulus contrast. Thus the gain of the system is allowed to change at both monocular and binocular sites (Fig. $1 B)$. If there are $m$ contrast levels tested, there will be $m * 4$ parameters for this model. In the monocular gain control only model, there are again four parameters describing the transfer characteristics at the highest (reference) contrast condition. How- 
A

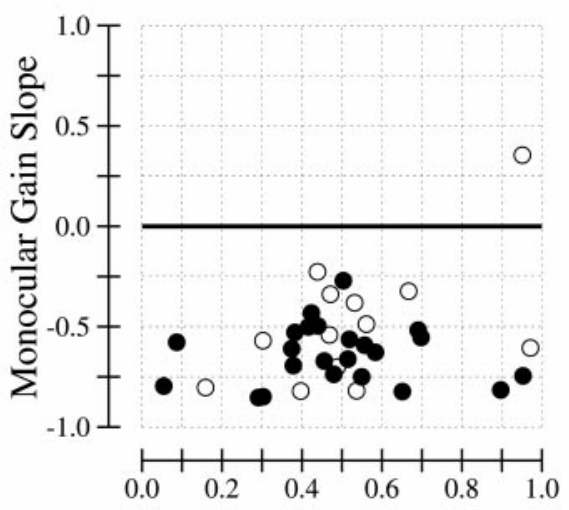

B

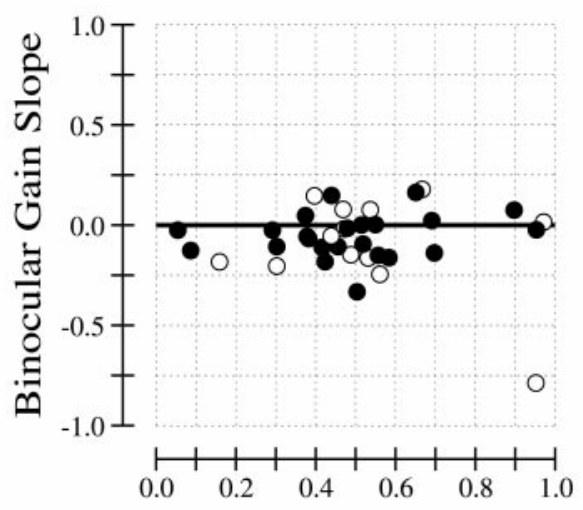

C

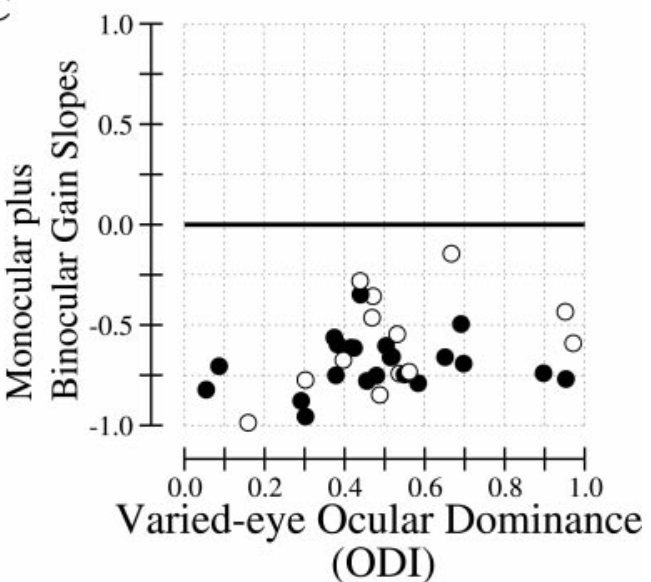

Figure 10. Analysis of the relationship between the ocular dominance index $(O D I)$ of a neuron and the magnitude of contrast-dependent gain reductions. Data are shown for experiments with a high contrast $(50 \%$, solid circles) or a lower contrast (4-10\%, unfilled circles) in the fixed eye. The horizontal line at 0 corresponds to the absence of gain control, whereas gain values of -1 correspond to $100 \%$ gain control. Correlations between $O D I$ and monocular gain slope or binocular gain slope are not significant $(p>0.05)$, regardless of whether the high- and low-contrast conditions are considered separately or together.

ever, at other contrasts, only the gain and phase at the monocular site (Fig. $1 B$ ) are allowed to change, giving a total of two parameters for each additional contrast condition, and hence a grand total of $4+m^{*} 2$ parameters. Likewise, in the binocular gain control only model, only the gain and phase parameters at the binocular site are allowed to change, again giving a grand total of $4+m^{*} 2$ parameters. The results are shown in Table 1 .

What is perhaps surprising here is that the average percentage residual error is quite low for both the monocular gain control only model $(6.3 \%)$ and the binocular gain control only model $(8.2 \%)$, compared with a value of $4.9 \%$ for the standard model. In terms of percentage residual error, the monocular gain control model clearly outperforms the binocular gain model (two-sided paired $t$ test, $t=5.4, p<0.001)$. However, if gain control is primarily monocular, as we have asserted, how can the binocular gain control only model account for so much of the variance in the data? First, even a model without any gain control at all should be able to account entirely for most of the contribution of the fixed eye to the response, which does not change much with contrast. Second, a model without any gain control should account for part of the contribution of the varied eye. Third, any changes in the monocular gain parameter for the varied eye can be emulated by a binocular gain control mechanism, although this comes at the expense of altering the gain in the other eye as well. So roughly speaking, a binocular gain control mechanism could account for $\sim 50 \%$ of the effect of a monocular gain control mechanism. Consistent with this point of view is the fact that the average binocular gain slope of -0.32 for the binocular gain model is approximately one-half of the average monocular gain slope of -0.69 for the monocular model; if gain control were predominately binocular, the opposite relationship would hold. Qualitatively, the binocular gain control model performs poorly; Figures $11 F$ and $12 F$ provide two examples.

Other gain control models involve variations in the static output nonlinearity (Fig. 1B), which is modeled parametrically (Eq. 1). The instantaneous effect of the nonlinearity is as follows. First, an offset value $T$ is subtracted from the signal. Second, if the signal is negative, it is set to 0 ; otherwise, it is raised to the exponent $n$. In the "standard model," $T=0$ and $n=2$. In the "standard model + free exponent," the exponent parameter $n$ is freed, although both this parameter and the binocular phase parameter are required to remain constant with changing contrast. (The phase parameter is constrained to reduce the total number of model parameters.) In the "free exponent model," the exponent $n$ is allowed to change with contrast, whereas both binocular phase and gain are required to remain constant. Thus, in this model, any binocular effects of gain control are mediated via a contrastdependent change in the exponent. The "standard + offset" and "additive offset" models are defined similarly, except that it is the offset parameter $T$ rather than the exponent that is allowed to change.

Finally, the "parametric variance" model is the same as the standard model, except that for the sake of determining $\chi^{2}$, the nonlinear regression algorithm uses an empirically constrained parametric model of response variance.

In models in which the monocular gain parameter is free to vary with contrast, the average monocular gain slope is large and negative, ranging in value from -0.55 to -0.62 , in all but one case. The exceptional case is the monocular gain control only model, with an average monocular gain slope of -0.69 . In models in which the binocular gain was free to vary with contrast, the average binocular gain slope was relatively small, ranging from -0.07 to -0.13 in all but one case. The exceptional case is the binocular gain control model only model, with an average binocular gain slope of -0.32 . In the exponent and offset models, the output nonlinearity, rather than the binocular linear filter, serves as the potential site of binocular gain control. In these models, a large monocular gain slope is observed (Table 1), but only modest effects of contrast on the exponent or offset parameter are seen (data not shown). Altogether, these data confirm that gain control is operating largely at a monocular site. 


\begin{tabular}{lllll}
\hline Table 1. Variants of the gain control model, as described in the text & & \\
& $\begin{array}{l}\text { \% Residual } \\
\text { error }\end{array}$ & $\begin{array}{l}\text { Monocular } \\
\text { gain slope }\end{array}$ & $\begin{array}{l}\text { Binocular } \\
\text { gain slope }\end{array}$ & $\begin{array}{l}\text { Number of } \\
\text { parameters }\end{array}$ \\
\hline Standard model & $4.9 \pm 2.8$ & $-0.58 \pm 0.23$ & $-0.07 \pm 0.17$ & 20 \\
$\quad$ +Free exponent & $4.5 \pm 2.7$ & $-0.55 \pm 0.24$ & $-0.13 \pm 0.18$ & 17 \\
$\quad$ Free offset & $4.6 \pm 2.7$ & $-0.56 \pm 0.25$ & $-0.11 \pm 0.16$ & 17 \\
Monocular gain only & $6.3 \pm 3.3$ & $-0.69 \pm 0.26$ & & 12 \\
Binocular gain only & $8.2 \pm 2.9$ & & $-0.32 \pm 0.20$ & 12 \\
Exponent model & $4.5 \pm 2.6$ & $-0.58 \pm 0.21$ & & 17 \\
Additive offset model & $4.8 \pm 2.9$ & $-0.62 \pm 0.26$ & & 17 \\
Parametric variance & $6.5 \pm 4.0$ & $-0.55 \pm 0.24$ & $-0.08 \pm 0.16$ & 20
\end{tabular}

Mean values $( \pm 1$ SD) are shown. The number of model parameters is shown for experiments with five rather than six contrast levels in the varied eye. Data from high-contrast experiments $(n=23)$ are combined with results from low-contrast experiments $(n=13)$. Percentage residual error equals $100 \% \times s s e / s s r$, where $s s r$ denotes the sum-of-squares of the neural response amplitudes, and sse denotes the sum-of-squares of the deviations of the neural responses from the fits produced by the model.

\section{A. Cubic Spline}

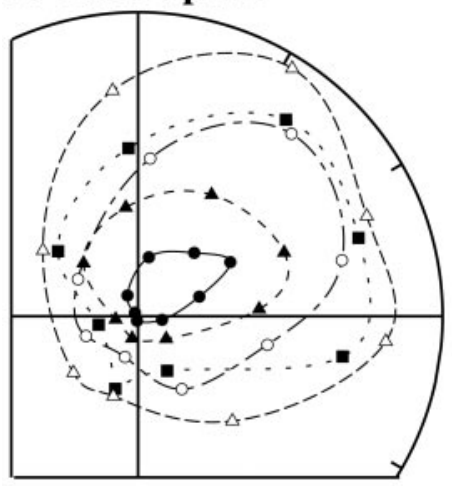

D. Additive Offset Model

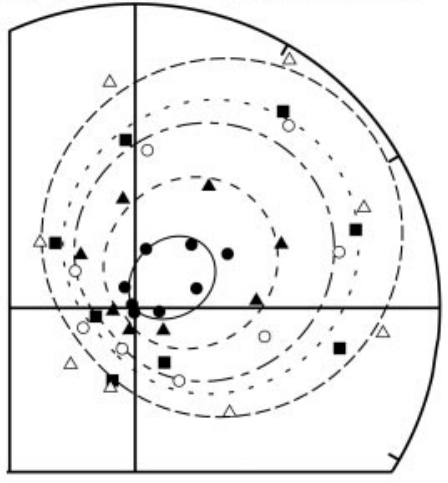

B. Standard Model

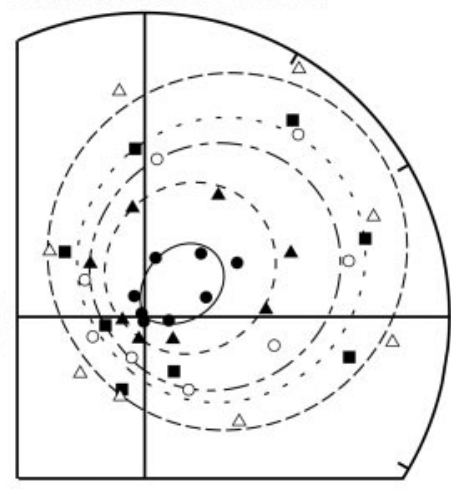

E. Monocular Gain Only

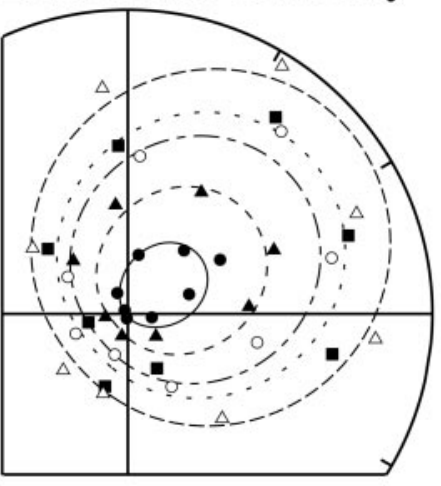

C. Exponent Model

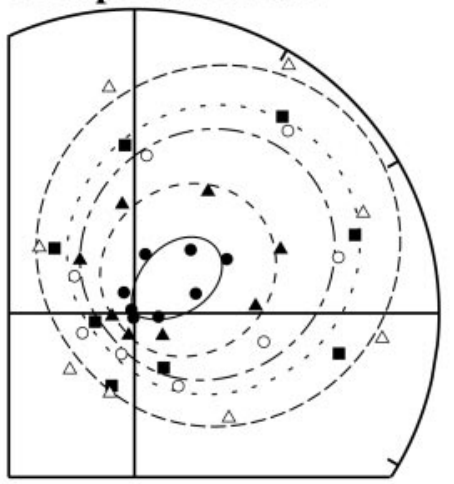

F. Binocular Gain Only

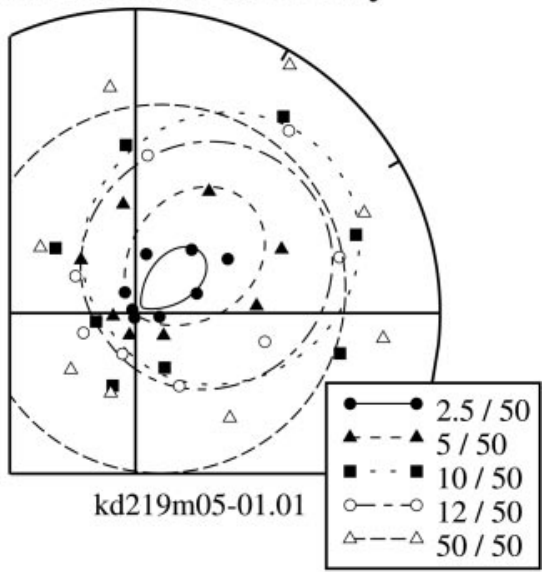

Figure 11. Variations on the gain control model. Each plot shows the fit (solid-line curves) of various models to data (symbols) collected from a binocular simple cell; the data are identical in the various plots. $A$, Cubic splines provide a convenient way of looking at the raw data. The percentage residual error is by definition $0 . B$, In the standard model ( $2.0 \%$ residual error), the phase and gain parameters for both eyes are allowed to change with contrast; the exponent and offset are fixed at 2 and 0 , respectively. $C$, In the exponent model (1.8\% residual error), both the monocular gain and the output exponent are allowed to vary with contrast; thus, any binocular gain control effects must be mediated by a change in exponent. $D$, In the offset model (1.9\% residual error), the exponent is fixed at 2 , and the additive offset parameter serves as the potential site of gain control. $E, F$, In these models, gain control is required to operate exclusively at a monocular or binocular site; the percentage residual error is 2.1 and $6.3 \%$, respectively. Note that most of the models (models $B-E$ ) allow gain control to operate at a monocular site; the output from these models is qualitatively similar and produces a reasonable fit to the data. The one model without monocular gain control (model $F$ ) produces a comparatively poor fit.

Because of the relatively weak effects of binocular gain control, it is not possible for us to distinguish between the exponent, the offset, and the binocular gain parameter as potential sites of the binocular gain control mechanism. We find that models with freed exponent parameters (e.g., exponent model or standard model + exponent) generally perform better than models without a free exponent parameter (e.g., offset model or standard model + offset); also, the geometry of the curves produced by the 


\section{A. Cubic Spline}

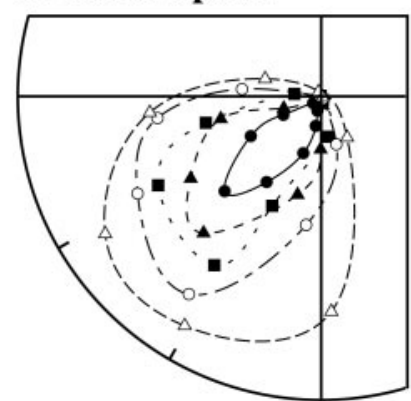

D. Additive Offset Model
B. Standard Model
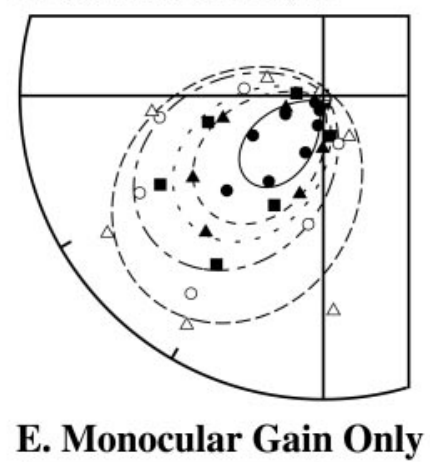

\section{Exponent Model}

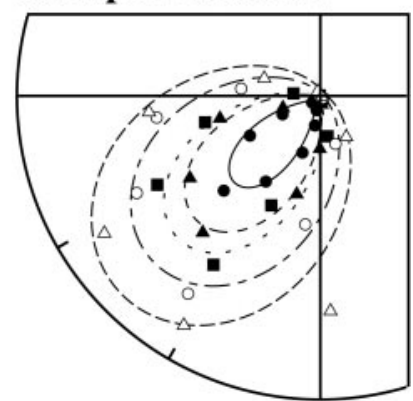

F. Binocular Gain Only

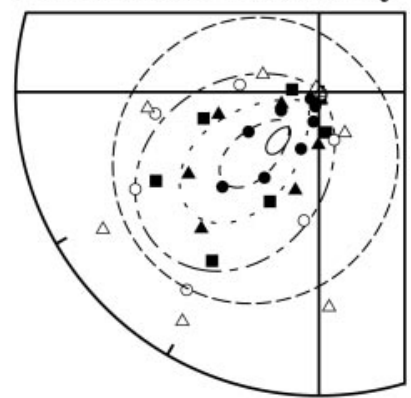

Figure 12. Model fits for a second simple cell, plotted in the same format as Figure 11. The best performing models are those that allow the monocular gain parameter to change as a function of contrast $(B-E$; percentage residual error $=1.6,1.3,1.3$, and 2.0 , respectively). Compared with the output of the standard model $(B)$, in which the output exponent is fixed at a value of 2, the elliptical response profiles produced by the exponent model ( $C$ ) are narrow and elongated; this is attributable to the higher values of exponent parameter (2.6-3.0), which give the best fit for this cell. Overall, however, the performance of models $B-E$ is similar. On the other hand, the binocular gain control model performs poorly $(F$; percentage residual error $=7.92 \%)$. The worst fits are evident at the extremes of the contrast range tested: at $2.5 \%$ contrast, the response curve is too small (solid-line curve), whereas at $50 \%$ contrast the response curve is too large (exterior dashed-line curve). This pattern of error is expected if gain control is mediated at a monocular site.

various models depends somewhat on the form of the output nonlinearity. Nonetheless, the variations that we examined in the functional form of the output nonlinearity have relatively minor effects on the qualitative behavior of the models (e.g., Fig. 12). This suggests that more sophisticated stimuli than those we used are needed to determine the detailed functional form of the output nonlinearity.

A final point concerns the possible effect of varied-eye contrast on the monocular filter parameters for the fixed eye. In the standard model, varied-eye contrast can affect the binocular filter but not the filter of the fixed eye. However, it is possible that an increase in contrast in one eye specifically suppresses responses from the other eye. Thus, in the "interocular suppression" model, the filter of the fixed eye, rather than the binocular filter, is allowed to change as a function of varied-eye contrast. The difference between the standard and the interocular suppression models is mathematically trivial: it amounts to a slightly different way of parceling the gain effects into monocular, binocular, and interocular components. In the interocular suppression model, the average monocular gain slope in the varied eye is -0.65 . This equals the sum of the average monocular and binocular gain slopes from the standard model. The average monocular gain slope in the fixed eye is -0.07 . This equals the average binocular gain slope from the standard model and reflects the magnitude of interocular suppression. Thus, in the interocular suppression model, there is a weak interocular effect and once again a strong monocular effect.

\section{DISCUSSION}

In this study, we examined a basic feature of contrast encoding in simple cells: contrast gain decreases as stimulus contrast is increased. When simple cells are stimulated dichoptically over a range of contrast levels, the contrast-dependent reductions in contrast gain are associated with a monocular site of processing. The presence or absence of a grating in one eye (the varied eye) is found to have relatively little effect on the contrast gain of the other eye (the fixed eye), regardless of whether the contrast level in the fixed eye is low (4-10\%) or high $(50 \%)$.

These findings raise a number of questions about the functional organization of contrast encoding. First, what is the physiological site of the monocular gain reductions? The monocular gain reductions might arise at a subcortical site. However, published data on geniculate contrast-response functions are limited in scope, and it is unclear how neural activity in the LGN is transduced into cortical response amplitudes. Thus, one cannot rule out a major involvement of cortical mechanisms. For example, studies of cross-orientation inhibition (DeAngelis et al., 1992; Walker et al., 1998) provide evidence of a suppressive nonlinearity at a monocular stage of cortical processing. Our study therefore underscores the need for further experimental comparisons of contrast encoding at different stages of visual processing.

A second question concerns the mechanism underlying the gain reductions. One hypothesis, shown in Figure $1 B$, is that the monocular and binocular gain parameters of the model are affected by the action of one or more gain control systems. Note 
that, by definition, a gain control effect is a dynamic contrastdependent nonlinearity. Although a static front-end nonlinearity may contribute to the monocular gain reductions, a major role for static nonlinearites seems to be ruled out by the quasi-sinusoidal waveforms of the neural responses (Fig. 2). Indeed, if static nonlinearities accounted for the strong gain reductions that we have observed, then increases in stimulus contrast would have progressively and dramatically distorted the response waveforms, with nearly square-wave responses at the highest contrasts. Such an effect was not observed.

\section{Binocular gain effects}

We report that the signal strength of one eye is relatively unaffected by the contrast level of the stimulus presented to the other eye. Indeed, the binocular gain parameter of the model does not change much as a function of stimulus contrast. [Although the model formally requires that interocular effects be mediated at a binocular site (Fig. $1 B, G_{\mathrm{B}}$ ), requiring that the effects be mediated at the monocular channel for the fixed eye (Fig. $1 B, G_{\mathrm{F}}$ ) leads to a quantitatively similar result.] It is interesting to compare this result with studies of cross-orientation inhibition, where the response to a grating of optimal spatial orientation is suppressed by a grating of nonoptimal orientation. These effects are much stronger when the optimal and orthogonal gratings are present in the same eye rather than in opposite eyes (DeAngelis et al., 1992; Walker et al., 1998). This has lead to the proposal of monocular inhibition at an early stage of cortical processing (Walker et al., 1998) and is consistent with our finding of a stronger role for monocular compared with binocular gain control. Nonetheless, there is a substantial interocular crossorientation effect (Sengpiel et al., 1995; Walker et al., 1998); on average, the crossed grating in one eye produces a $\sim 19.9 \pm 29 \%$ reduction to the optimal grating in the second eye (Walker et al., 1998). It has been proposed that cross-orientation suppression reflects an inhibitory process that is functionally present at all stimulus orientations but becomes experimentally confounded with the excitatory effects of a grating at the optimal orientation (Sengpiel et al., 1995). Thus, studies of cross-orientation inhibition should be relevant to our results, although we are using optimally oriented gratings in both eyes. We find that the presentation of a grating in the varied eye reduces the binocular gain parameter by a factor of 1.2. Accounting for the half-squaring nonlinearity of the model, this crudely translates into a reduction of response rate by a factor of $1-1 / 1.2^{2}$, or $31 \%$. So although the binocular gain effects that we report are small in comparison to the monocular gain effects, they are not inconsistent with a significant effect of binocular gain control on neural response rate.

Although not directly relevant to this study, there are other aspects of cortical visual encoding for which a distinction between monocular and binocular mechanisms has been made.

Unlike cross-orientation inhibition, surround suppression effects, which are elicited by stimulating regions surrounding the classical receptive field, are almost equal with monoptic and dichoptic presentations (DeAngelis et al., 1994). A number of investigators have examined the interocular effects of neuronal adaptation to prolonged stimulation, although mixed conclusions have been reached, with some studies finding strong interocular effects (Maffei et al., 1986; Marlin et al., 1991) and others showing much weaker effects (Hammond and Mouat, 1988). In this regard, it should be noted that our finding of weak binocular interaction applies to the $4 \mathrm{sec}$ time window during which each stimulus was presented in our study. Short-term adaptation effects in the striate cortex of the cat fall partly outside this time window. For example, an average of $17.0 \mathrm{sec}$ is required for simple cell responses to fall to $33 \%$ of their initial peak value (Albrecht et al., 1984). It has been suggested that the stimulus-mediated adjustments in response gain in the visual cortex occur in rapid, moderate, and slow time scales [100 msec, 5-10 sec, and minutes to hours (Bonds, 1991)]. Our results clearly apply to rapid effects, and to short-term $(5-10 \mathrm{sec})$ effects, but no conclusion can be reached about long-term adaptation effects.

In sum, there is evidence for both monocular and binocular suppressive mechanisms in primary visual cortex. Our results, however, suggest that monocular gain control plays the major role in determining the contrast-response relationship for binocular simple cells under dichoptic viewing conditions.

\section{Other studies of monocular gain control}

The finding of strong monocular effects is consistent with previous studies in the cat (Freeman and Ohzawa, 1990; Ohzawa and Freeman, 1994) and macaque (Smith et al., 1997b). With contrast in one eye fixed, these studies found that the depth of modulation of relative-phase tuning curves remained relatively stable as a function of varied-eye contrast, suggesting a robust monocular gain control system.

\section{Limitations of our gain control model}

To some extent, the model of the binocular simple cell used in this study is well specified: the simple cell is a linear mechanism with a static output nonlinearity. However, the third component of the model, the gain control mechanism, is not fully specified, because the model makes no assertions about the tuning characteristics or the transient dynamics of this mechanism. Therefore, the model cannot be used to predict the response of the system to an arbitrary stimulus. Rather, the model describes the timeaveraged effect of gain control during the $4 \mathrm{sec}$ of stimulus presentation. The model and our experimental design thus have the following constraints: (1) only effects of gain control within the $4 \mathrm{sec}$ stimulus window are reflected in the data, and (2) the detailed dynamics of the gain control system within this window have not been investigated.

\section{Perceptual consequences of unequal contrast ratios}

In all, the properties of the gain control system seem well suited to promoting the stability of binocular interactions in the face of unequal contrast levels in the two eyes. The strong monocular gain control system minimizes the effect of altered monocular contrast on binocular mechanisms of the striate cortex, whereas the limited nature of interocular suppressive effects prevents increased signal strength in one eye from abolishing the signal from the other eye. As a corollary, one might think that increasing signal strength in one eye will improve the ability of an individual neuron to encode either monocular or binocular features of the stimulus. From a statistical point of view, this question is complicated by the fact that neural response variability increases with mean response amplitude (Dean, 1981; Tolhurst et al., 1983; Anzai et al., 1995; and data not shown). Nonetheless, our findings suggest the hypothesis that interocular mismatches in contrast do not strongly or consistently degrade the sensitivity of simple cells to binocular features in an image.

In general, it is unclear whether unequal contrast ratios disrupt the sensitivity of the earliest binocular mechanisms in human vision. Unequal interocular contrast ratios disrupt stereomatching (Smallman and McKee, 1995), interfere with the transient stereopsis system (Schor et al., 1998), and degrade stereoacuity 
(Halpern and Blake, 1988; Legge and Gu, 1989; Schor and Heckmann, 1989) at low spatial frequencies (Cormack et al., 1997). The effect of contrast on stereoacuity has been described as "paradoxical" in the sense that an increase in physical signal strength results in a loss of sensitivity. The model of Kontsevich and Tyler (1994) accounts for the loss of stereoacuity in terms of mutual inhibition between monocular channels before the computation of disparity by early binocular mechanisms. This suggests that early binocular mechanisms in the human visual system exhibit stronger interocular suppression than we have observed in simple cells of the striate cortex of the cat. An alternative hypothesis is that "contrast paradox" effects reflect rivalry mechanisms that follow an initial binocular processing stage. This could include rivalry among channels that differ in the interocular contrast ratio that they prefer. Degradation of stereo vision would be expected if unequal contrast ratios give monocular mechanisms a competitive advantage.

In any case, it is interesting to note that unequal contrast ratios have little effect on human stereoacuity at high spatial frequencies (Cormack et al., 1997) or on vertical fusion limits (Schor and Heckmann, 1989). Such findings could reflect the action of a monocular gain control mechanism, which stabilizes the processing of binocular information in the presence of unequal levels of monocular contrast.

\section{REFERENCES}

Albrecht DG, Geisler WS (1991) Motion sensitivity and the contrastresponse function of simple cells in visual cortex. Vis Neurosci 7:531-546.

Albrecht DG, Hamilton DB (1982) Striate cortex of monkey and cat: contrast response function. J Neurophysiol 48:217-237.

Albrecht DG, Farrar SB, Hamilton DB (1984) Spatial contrast adaptation characteristics of neurones recorded in the cat's visual cortex. J Physiol (Lond) 347:713-739.

Anderson PA, Movshon JA (1989) Binocular combination of contrast signals. Vision Res 29:1115-1132.

Anzai A, Bearse Jr MA, Freeman RD, Cai D (1995) Contrast coding by cells in the cat's striate cortex: monocular vs. binocular detection. Vis Neurosci 12:77-93.

Bauman LA, Bonds AB (1991) Inhibitory refinement of spatial frequency selectivity in single cells of the cat striate cortex. Vision Res 31:933-944.

Benardete EA, Kaplan E, Knight BW (1992) Contrast gain control in the primate retina: $\mathrm{P}$ cells are not $\mathrm{X}$-like, some $\mathrm{M}$ cells are. Vis Neurosci 8:483-486.

Bonds AB (1991) Temporal dynamics of contrast gain in single cells of the cat striate cortex. Vis Neurosci 6:239-255.

Carandini M, Heeger DJ (1994) Summation and division by neurons in primate visual cortex. Science 264:1333-1336.

Carandini M, Heeger DJ, Movshon JA (1993) Amplitude and phase of contrast responses in LGN and V1. Soc Neurosci Abstr 19:628.

Carandini M, Heeger DJ, Movshon JA (1997) Linearity and normalization in simple cells of the macaque primary visual cortex. J Neurosci 17:8621-8644.

Carandini M, Heeger DJ, Movshon JA (1999) Linearity and gain control in V1 simple cells. In: Cerebral cortex, Vol 13: models of cortical function (Ulinski PS, Jones EG, Peters A, eds), pp 401-443. New York: Kluwer Academic Plenum.

Cormack LK, Stevenson SB, Landers DD (1997) Interactions of spatial frequency and unequal monocular contrasts in stereopsis. Perception 26:1121-1136.

Dean AF (1981) The variability of discharge of simple cells in the cat striate cortex. Exp Brain Res 44:437-440.

Dean AF, Tolhurst DJ (1986) Factors influencing the temporal phase of response to bar and grating stimuli for simple cells in the cat striate cortex. Exp Brain Res 62:143-151.

DeAngelis GC, Robson JG, Ohzawa I, Freeman RD (1992) Organiza- tion of suppression in receptive fields of neurons in cat visual cortex. J Neurophysiol 68:144-163.

DeAngelis GC, Ohzawa I, Freeman RD (1993) Spatiotemporal organization of simple-cell receptive fields in the cat's striate cortex. II. Linearity of temporal and spatial summation. J Neurophysiol 69:1118-1135.

DeAngelis GC, Freeman RD, Ohzawa I (1994) Length and width tuning of neurons in the cat's primary visual cortex. J Neurophysiol 71:347-374.

DeValois RL, Tootell RBH (1983) Spatial-frequency-specific inhibition in cat striate cortex cells. J Physiol (Lond) 336:359-376.

Ferster D (1988) Spatially opponent excitation and inhibition in simple cells of the cat visual cortex. J Neurosci 8:1172-1180.

Freeman RD, Ohzawa I (1990) On the neurophysiological organization of binocular vision. Vision Res 30:1661-1676.

Halpern L, Blake RR (1988) How contrast affects stereoacuity. Perception 17:483-495.

Hammond P, Mouat GSV (1988) Neural correlates of motion aftereffects in cat striate cortical neurones: interocular transfer. Exp Brain Res 72:21-28.

Heeger DJ (1992a) Half-squaring in responses of cat striate cells. Vis Neurosci 9:427-443.

Heeger DJ (1992b) Normalization of cell responses in cat striate cortex. Vis Neurosci 9:181-197.

Heeger DJ (1993) Modeling simple-cell direction selectivity with normalized, half-squared linear operators. J Neurophysiol 70:1885-1898.

Hubel DH, Wiesel TN (1962) Receptive fields, binocular interaction and functional architecture in the cat's visual cortex. J Physiol (Lond) 160:106-154.

Jones JP, Palmer LA (1987) An evaluation of the two-dimensional Gabor filter model of simple receptive fields in cat striate cortex. J Neurophysiol 58:1233-1258.

Kontsevich LL, Tyler CW (1994) Analysis of stereo thresholds for stimuli below 2.5 cpd. Vision Res 34: 2317-2329.

Legge GE, Gu Y (1989) Stereopsis and contrast. Vision Res 29:989-1004.

Levick WR (1972) Another tungsten microelectrode. Med Biol Eng 10:510-515.

Macy A, Ohzawa I, Freeman RD (1982) A quantitative study of the classification and stability of ocular dominance in the cat's visual cortex. Exp Brain Res 48:401-408.

Maffei L, Berardi N, Bisti S (1986) Interocular transfer of adaptation after effect in neurons of area 17 and 18 of split chiasm cats. J Neurophysiol 55:966-976.

Marlin SG, Douglas RM, Cynader MS (1991) Position-specific adaptation in simple cell receptive fields of the cat striate cortex. J Neurophysiol 66:1769-1784.

Morrone MC, Burr DC, Maffei L (1982) Functional implications of cross-orientation inhibition of cortical visual cells. I. Neurophysiological evidence. Proc R Soc Lond B Biol Sci 216:335-354.

Movshon JA, Thompson ID, Tolhurst DJ (1978) Spatial summation in the receptive fields of simple cells in the cat's striate cortex. J Physiol (Lond) 283:53-77.

Nestares O, Heeger DJ (1997) Modeling the apparent frequency-specific suppression in simple cell responses. Vision Res 37:1535-1543.

Ohzawa I, Freeman RD (1986) The binocular organization of simple cells in the cat's visual cortex. J Neurophysiol 56:221-242.

Ohzawa I, Freeman RD (1994) Monocular and binocular mechanisms of contrast gain control. In: Computational vision based on neurobiology (Lawton TB, ed), pp 43-51. Bellingham, WA: SPIE.

Ohzawa I, Sclar G, Freeman RD (1982) Contrast gain control in the cat visual cortex. Nature 298:266-268.

Ohzawa I, Sclar G, Freeman RD (1985) Contrast gain control in the cat's visual system. J Neurophysiol 54:651-667.

Ohzawa I, DeAngelis GC, Freeman RD (1996) Encoding of binocular disparity by simple cells in the cat's visual cortex. J Neurophysiol 75:1779-1805.

Press WH, Teukolsky SA, Vetterling WT, Flannery BP (1992) Numerical recipes in C, Ed 2. Cambridge, UK: Cambridge UP.

Schor C, Heckmann T (1989) Interocular differences in contrast and spatial frequency: effects on stereopsis and fusion. Vision Res 29:837-847. 
Schor CM, Edwards M, Pope DR (1998) Spatial-frequency and contrast tuning of the transient-stereopsis system. Vision Res 38:3057-3068.

Sclar G, Freeman RD (1982) Orientation selectivity in the cat's striate cortex is invariant with stimulus contrast. Exp Brain Res 46:457-461.

Sclar G, Ohzawa I, Freeman RD (1985) Contrast gain control in the kitten's visual system. J Neurophysiol 54:668-675.

Sengpiel F, Blakemore C (1994) Interocular control of neuronal responsiveness in cat visual cortex. Nature 368:847-850.

Sengpiel F, Blakemore C, Harrad R (1995) Interocular suppression in the primary visual cortex: a possible neural basis of binocular rivalry. Vision Res 35:179-195.

Shapley RM, Victor JD (1978) The effect of contrast on the transfer properties of cat retinal ganglion cells. J Physiol (Lond) 285:275-298.

Shapley R, Enroth-Cugell C (1984) Visual adaptation and retinal gain controls. Prog Retinal Res 3:263-343.

Skottun BC, De Valois RL, Grosof DH, Movshon JA, Albrecht DG, Bonds AB (1991) Classifying simple and complex cells on the basis of response modulation. Vision Res 31:1079-1086.

Smallman HS, McKee SP (1995) A contrast ratio constraint on stereo matching. Proc R Soc Lond B Biol Sci 260:265-271.
Smith III EL, Chino YM, Ni J, Ridder III WH, Crawford MLJ (1997a) Binocular spatial phase tuning characteristics of neurons in the macaque striate cortex. J Neurophysiol 78:351-365.

Smith III EL, Chino Y, Ni J, Cheng H (1997b) Binocular combination of contrast signals by striate cortical neurons in the monkey. J Neurophysiol 78:366-382.

Tolhurst DJ, Heeger DJ (1997a) Contrast normalization and a linear model for the directional selectivity of simple cells in cat striate cortex. Vis Neurosci 14:19-25.

Tolhurst DJ, Heeger DJ (1997b) Comparison of contrast-normalization and threshold models of the responses of simple cells in cat striate cortex. Vis Neurosci 14:293-309.

Tolhurst DJ, Movshon JA, Dean AF (1983) The statistical reliability of signals in single neurons in cat and monkey visual cortex. Vision Res 23:775-785.

Victor JD (1987) The dynamics of the cat retinal X cell centre. J Physiol (Lond) 386:219-246.

Walker GA, Ohzawa I, Freeman RD (1998) Binocular cross-orientation suppression in the cat's striate cortex. J Neurophysiol 79:227-239. 